\title{
Nanocomposites Containing Keggin Anions Anchored on Pyrazine-Based Frameworks for Use as Supercapacitors and Photocatalysts
}

Nana Du $u^{a, b}$, Lige Gong ${ }^{a, b}$, Lingyu Fan ${ }^{a, b}$, Kai Yu ${ }^{a, b, *}$, Huan Luo ${ }^{a, b}$, Shengjie Pang ${ }^{a, b}$, Jiaqian Gao ${ }^{a, b}$, Zhuwu Zheng ${ }^{a, b}$, Jinghua Lv ${ }^{a}$, Baibin Zhou ${ }^{a, b, *}$

a Key Laboratory for Photonic and Electronic Band gap Materials, Ministry of Education, Harbin Normal University, Harbin 150025, People's Republic of China.

b Key Laboratory of Synthesis of Functional Materials and Green Catalysis, Colleges of Heilongjiang Province, Harbin Normal University, Harbin, 150025,People's Republic of China

E-mail: hlyukai188@163.com, zhou_bai_bin@163.com

\section{Materials and Characterization measurements}

All the chemic reagents were commercially purchased, and not further purified during the use process. Elemental analyses $(\mathrm{C}, \mathrm{H}$, and $\mathrm{N})$ were measured on a Perkin-Elmer $2400 \mathrm{CHN}$ elemental analyzer. B, Si, P, Ag, Mo and W were detected on a PLASMA-SPEC (I) ICP atomic emission spectrometer. The IR spectrum was measured with an Alpha Centaurt FT/IR pectrophotometer in the range of $400-4000 \mathrm{~cm}^{-1}$ using $\mathrm{KBr}$ pellets. The results of TG analyses were obtained by a Perkin-Elmer TGA7 analyzer and the range of temperature was from 20 to $800{ }^{\circ} \mathrm{C}$ 
under $\mathrm{O}_{2}$. XRD patterns were performed on RigakuDmax 2000 X-ray diffractometer with graphite monochromatized $\mathrm{Cu} \mathrm{K} \alpha$ radiation $(\lambda=0.154 \mathrm{~nm})$ and $2 \theta$ ranging from 5 to $50^{\circ}$. The scanning electron microscopy (SEM) images were measured by a Hitachi SU70 SEM coupled with an energy-dispersive X-ray (EDX) detector. The transmission electron microscope (TEM) images were captured by Tecnai G2F20. UV-Vis-NIR absorption spectroscopy $\left(\mathrm{BaSO}_{4}\right.$ pellets) was collected with a Cary 500 spectrophotometer.

\section{X-ray crystallography}

Crystal data for compounds 1-3 were obtained by a Bruker SMART CCD detector with graphite monochromatic $\operatorname{MoK} \alpha$ radiation $(\lambda=0.71073 \AA)$. The structures were solved by direct methods and refined by full-matrix least-squares on F2 using the Olex2program. All the non-hydrogen atoms were refined anisotropically. All the hydrogen atoms were set idealized positions and refined. Crystallographic data and structure refinements for compounds 1-3 are summarized in Table S1. Selected bond lengths $(\AA)$ and angles $\left(^{\circ}\right)$ for compounds 1-3 are listed in Table S2-S4.

Table S1. Crystal Datas and Structure Refinements for 1, 2 and 3

\begin{tabular}{llll}
\hline Compound & $\mathbf{1}$ & $\mathbf{2}$ & $\mathbf{3}$ \\
\hline Chemical formula & $\mathrm{C}_{28} \mathrm{H}_{28} \mathrm{Ag}_{5} \mathrm{~N}_{14} \mathrm{O}_{40} \mathrm{BW}_{12}$ & $\mathrm{C}_{28} \mathrm{H}_{28} \mathrm{Ag}_{5} \mathrm{~N}_{14} \mathrm{O}_{42} \mathrm{SiW}_{12}$ & $\mathrm{C}_{12} \mathrm{H}_{12} \mathrm{Ag}_{2} \mathrm{~N}_{5} \mathrm{O}_{40} \mathrm{PMo}_{12}$ \\
Formula weight & 3956.89 & 4006.17 & 2264.26 \\
$T / \mathrm{K}$ & $296(2)$ & $296(2)$ & $296(2)$ \\
Crystal system & Triclinic & Monoclinic & Orthorhombic \\
Space group & $\mathrm{P}-1$ & $\mathrm{C} 2 / \mathrm{c}$ & $\mathrm{C}$ m c 21 \\
$a / \AA$ & $12.542(9)$ & $23.232(4)$ & $16.808(3)$ \\
$b / \AA$ & $15.311(13)$ & $20.543(4)$ & $18.827(3)$ \\
$c / \AA$ & $15.452(11)$ & $12.541(2)$ & $14.104(3)$ \\
$\alpha /{ }^{\circ}$ & $82.86(3)$ & 90.00 & 90.00 \\
$\beta /$ & $84.36(2)$ & $97.922(2)$ & 90.00
\end{tabular}




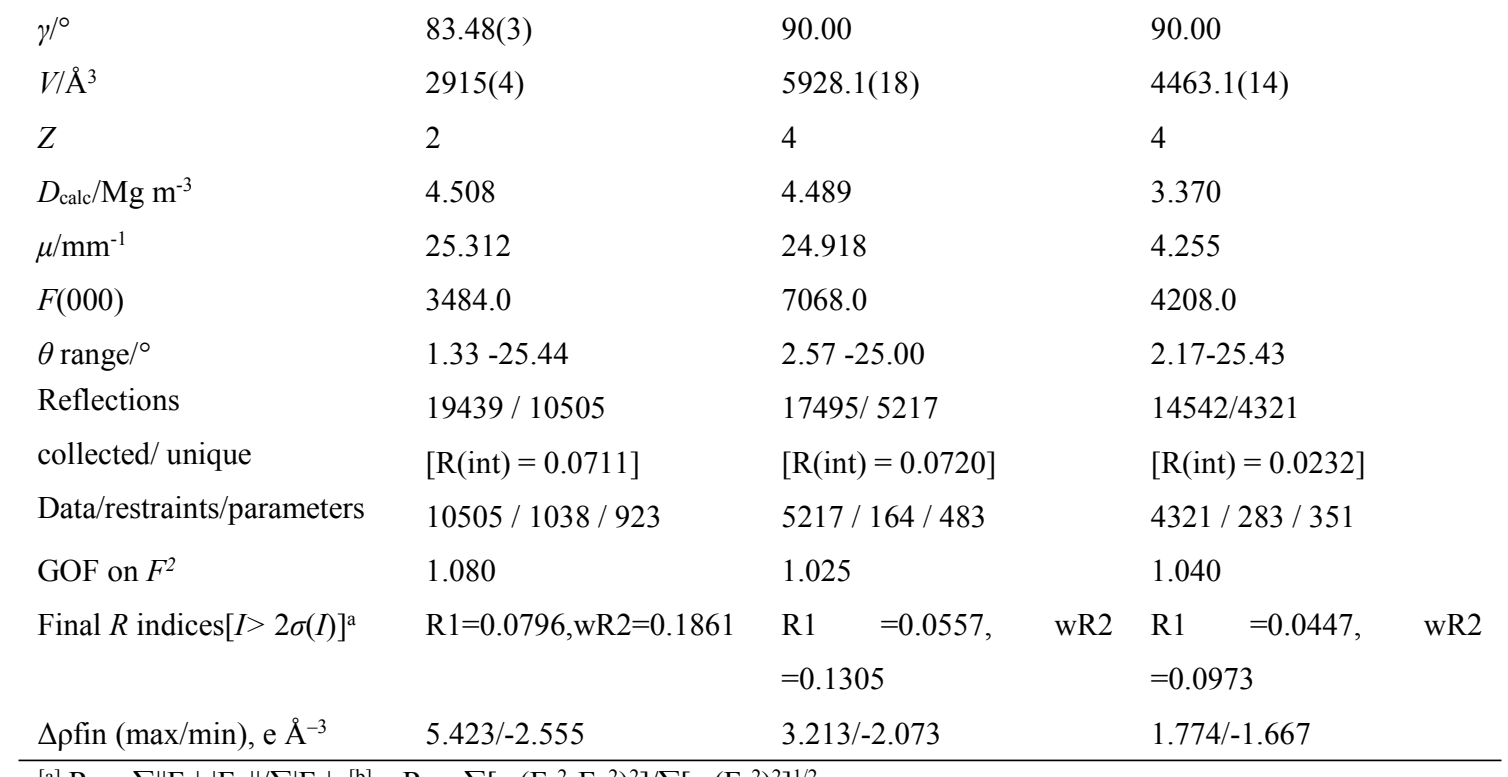

${ }^{[a]} \mathrm{R}_{1}=\sum|| \mathrm{F}_{0}|-| \mathrm{F}_{\mathrm{C}} \| / \sum\left|\mathrm{F}_{0}\right| ;{ }^{[\mathrm{b}]} \mathrm{wR}_{2}=\sum\left[\mathrm{w}\left(\mathrm{F}_{0}{ }^{2}-\mathrm{F}_{\mathrm{C}}{ }^{2}\right)^{2}\right] / \sum\left[\mathrm{w}\left(\mathrm{F}_{0}{ }^{2}\right)^{2}\right]^{1 / 2}$

Table S2 Selected bond lengths $(\AA)$ and bond angles $\left({ }^{\circ}\right)$ of compound 1

\begin{tabular}{|l|l|l|l|l|l|}
\hline $\mathrm{B}(1)-\mathrm{O}(37)$ & $1.55(6)$ & $\mathrm{B}(1)-\mathrm{O}(37) \# 1$ & $1.55(6)$ & $\mathrm{B}(1)-\mathrm{O}(38)$ & $1.50(5)$ \\
\hline $\mathrm{B}(1)-\mathrm{O}(38) \# 1$ & $1.50(5)$ & $\mathrm{B}(1)-\mathrm{O}(39)$ & $1.54(3)$ & $\mathrm{B}(1)-\mathrm{O}(39) \# 1$ & $1.54(3)$ \\
\hline $\mathrm{B}(2)-\mathrm{O}(36)$ & $1.47(5)$ & $\mathrm{B}(2)-\mathrm{O}(36) \# 1$ & $1.47(5)$ & $\mathrm{B}(2)-\mathrm{O}(40)$ & $1.46(5)$ \\
\hline $\mathrm{B}(2)-\mathrm{O}(40) \# 1$ & $1.46(5)$ & $\mathrm{B}(2)-\mathrm{O}(41)$ & $1.49(3)$ & $\mathrm{B}(2)-\mathrm{O}(41) \# 1$ & $1.49(3)$ \\
\hline $\mathrm{W}(1)-\mathrm{O}(10)$ & $1.82(3)$ & $\mathrm{W}(1)-\mathrm{O}(30)$ & $1.84(3)$ & $\mathrm{W}(1)-\mathrm{O}(31)$ & $1.86(3)$ \\
\hline $\mathrm{W}(1)-\mathrm{O}(32)$ & $1.88(3)$ & $\mathrm{W}(1)-\mathrm{O}(33)$ & $1.65(2)$ & $\mathrm{W}(1)-\mathrm{O}(39)$ & $2.34(3)$ \\
\hline $\mathrm{W}(2)-\mathrm{O}(3)$ & $1.68(2)$ & $\mathrm{W}(2)-\mathrm{O}(4)$ & $1.86(3)$ & $\mathrm{W}(2)-\mathrm{O}(22)$ & $1.86(3)$ \\
\hline $\mathrm{W}(2)-\mathrm{O}(28)$ & $1.84(3)$ & $\mathrm{W}(2)-\mathrm{O}(40)$ & $2.45(5)$ & $\mathrm{W}(2)-\mathrm{O}(41)$ & $2.39(3)$ \\
\hline $\mathrm{W}(2)-\mathrm{O}(0 A A)$ & $1.92(3)$ & $\mathrm{W}(3)-\mathrm{O}(8)$ & $1.68(2)$ & $\mathrm{W}(3)-\mathrm{O}(15)$ & $1.90(3)$ \\
\hline $\mathrm{W}(3)-\mathrm{O}(16)$ & $1.90(3)$ & $\mathrm{W}(3)-\mathrm{O}(26)$ & $1.84(3)$ & $\mathrm{W}(3)-\mathrm{O}(30)$ & $1.85(3)$ \\
\hline $\mathrm{W}(3)-\mathrm{O}(38)$ & $2.39(5)$ & $\mathrm{W}(3)-\mathrm{O}(39)$ & $2.43(3)$ & $\mathrm{W}(4)-\mathrm{O}(1)$ & $1.65(2)$ \\
\hline $\mathrm{W}(4)-\mathrm{O}(2)$ & $1.85(3)$ & $\mathrm{W}(4)-\mathrm{O}(4)$ & $1.91(3)$ & $\mathrm{W}(4)-\mathrm{O}(12)$ & $1.92(3)$ \\
\hline $\mathrm{W}(4)-\mathrm{O}(24)$ & $1.83(3)$ & $\mathrm{W}(4)-\mathrm{O}(36)$ & $2.37(5)$ & $\mathrm{W}(4)-\mathrm{O}(40)$ & $2.35(5)$ \\
\hline $\mathrm{W}(5)-\mathrm{O}(13)$ & $1.65(2)$ & $\mathrm{W}(5)-\mathrm{O}(23)$ & $1.93(3)$ & $\mathrm{W}(5)-\mathrm{O}(25)$ & $1.88(3)$ \\
\hline $\mathrm{W}(5)-\mathrm{O}(32)$ & $1.83(3)$ & $\mathrm{W}(5)-\mathrm{O}(34)$ & $1.85(3)$ & $\mathrm{W}(5)-\mathrm{O}(37)$ & $2.34(5)$ \\
\hline $\mathrm{W}(6)-\mathrm{O}(12)$ & $1.80(2)$ & $\mathrm{W}(6)-\mathrm{O}(14)$ & $1.64(2)$ & $\mathrm{W}(6)-\mathrm{O}(27)$ & $1.88(3)$ \\
\hline $\mathrm{W}(6)-\mathrm{O}(28)$ & $1.94(2)$ & $\mathrm{W}(6)-\mathrm{O}(29)$ & $1.86(3)$ & $\mathrm{W}(6)-\mathrm{O}(40)$ & $2.45(5)$ \\
\hline $\mathrm{W}(7)-\mathrm{O}(7)$ & $1.69(2)$ & $\mathrm{W}(7)-\mathrm{O}(17)$ & $1.86(3)$ & $\mathrm{W}(7)-\mathrm{O}(21)$ & $1.86(3)$ \\
\hline $\mathrm{W}(7)-\mathrm{O}(24)$ & $1.86(3)$ & $\mathrm{W}(7)-\mathrm{O}(36)$ & $2.50(5)$ & $\mathrm{W}(7)-\mathrm{O}(41)$ & $2.40(3)$ \\
\hline $\mathrm{W}(7)-\mathrm{O}(0 A A)$ & $1.85(3)$ & $\mathrm{W}(8)-\mathrm{O}(6)$ & $1.90(3)$ & $\mathrm{W}(8)-\mathrm{O}(10)$ & $1.93(3)$ \\
\hline $\mathrm{W}(8)-\mathrm{O}(11)$ & $1.63(3)$ & $\mathrm{W}(8)-\mathrm{O}(16)$ & $1.80(3)$ & $\mathrm{W}(8)-\mathrm{O}(23)$ & $1.85(3)$ \\
\hline $\mathrm{W}(8)-\mathrm{O}(37)$ & $2.31(5)$ & $\mathrm{W}(8)-\mathrm{O}(39)$ & $2.37(3)$ & $\mathrm{W}(9)-\mathrm{O}(9)$ & $1.69(2)$ \\
\hline $\mathrm{W}(9)-\mathrm{O}(15)$ & $1.89(3)$ & $\mathrm{W}(9)-\mathrm{O}(25)$ & $1.83(3)$ & $\mathrm{W}(9)-\mathrm{O}(31)$ & $1.90(3)$ \\
\hline $\mathrm{W}(9)-\mathrm{O}(35)$ & $1.88(3)$ & $\mathrm{W}(9)-\mathrm{O}(38)$ & $2.34(5)$ & $\mathrm{W}(10)-\mathrm{O}(5)$ & $1.68(3)$ \\
\hline $\mathrm{W}(10)-\mathrm{O}(6)$ & $1.86(3)$ & $\mathrm{W}(10)-\mathrm{O}(26)$ & $1.90(3)$ & $\mathrm{W}(10)-\mathrm{O}(34)$ & $1.87(3)$ \\
\hline $\mathrm{W}(10)-\mathrm{O}(35)$ & $1.82(3)$ & $\mathrm{W}(10)-\mathrm{O}(37)$ & $2.45(5)$ & $\mathrm{W}(10)-\mathrm{O}(38)$ & $2.44(6)$ \\
\hline & & & & & \\
\hline
\end{tabular}




\begin{tabular}{|c|c|c|c|c|c|}
\hline $\mathrm{W}(11)-\mathrm{O}(2)$ & $1.90(3)$ & $\mathrm{W}(11)-\mathrm{O}(17)$ & $1.86(2)$ & $\mathrm{W}(11)-\mathrm{O}(18)$ & $1.65(3)$ \\
\hline $\mathrm{W}(11)-\mathrm{O}(19)$ & $1.90(3)$ & $\mathrm{W}(11)-\mathrm{O}(27)$ & $1.85(3)$ & $\mathrm{W}(11)-\mathrm{O}(36)$ & $2.36(5)$ \\
\hline $\mathrm{W}(12)-\mathrm{O}(19)$ & $1.88(3)$ & $\mathrm{W}(12)-\mathrm{O}(20)$ & $1.69(2)$ & $\mathrm{W}(12)-\mathrm{O}(21)$ & $1.87(3)$ \\
\hline $\mathrm{W}(12)-\mathrm{O}(22)$ & $1.85(3)$ & $\mathrm{W}(12)-\mathrm{O}(29)$ & $1.86(3)$ & $\mathrm{W}(12)-\mathrm{O}(41)$ & $2.43(3)$ \\
\hline $\operatorname{Ag}(1)-\mathrm{N}(3)$ & $2.34(3)$ & $\operatorname{Ag}(1)-\mathrm{N}(9)$ & $2.33(3)$ & $\operatorname{Ag}(1)-\mathrm{N}(10)$ & $2.35(3)$ \\
\hline $\operatorname{Ag}(1)-\mathrm{N}(14)$ & $2.46(5)$ & $\operatorname{Ag}(2)-\mathrm{N}(11)$ & $2.19(3)$ & $\operatorname{Ag}(2)-\mathrm{N}(12)$ & $2.26(3)$ \\
\hline $\operatorname{Ag}(2)-\mathrm{N}(13)$ & $2.24(3)$ & $\operatorname{Ag}(3)-\mathrm{N}(2)$ & $2.19(3)$ & $\operatorname{Ag}(3)-\mathrm{N}(6)$ & $2.27(5)$ \\
\hline $\operatorname{Ag}(3)-\mathrm{N}(7)$ & $2.16(3)$ & $\operatorname{Ag}(4)-\mathrm{N}(1)$ & $2.22(3)$ & $\operatorname{Ag}(4)-\mathrm{N}(21)$ & $2.26(4)$ \\
\hline $\operatorname{Ag}(5)-\mathrm{N}(4)$ & $2.23(4)$ & $\operatorname{Ag}(5)-\mathrm{N}(5)$ & $2.19(3)$ & $\operatorname{Ag}(1)-O(5)$ & 2.77 \\
\hline $\mathrm{Ag}(1)-\mathrm{O}(20)$ & 2.80 & $\operatorname{Ag}(3)-O(14)$ & 2.68 & $\mathrm{Ag}(3)-\mathrm{O}(13)$ & 2.64 \\
\hline $\operatorname{Ag}(4)-O(9)$ & $2.45(3)$ & $\operatorname{Ag}(4)-O(18)$ & $2.51(3)$ & $\mathrm{Ag}(5)-\mathrm{O}(3)$ & $2.44(2)$ \\
\hline $\operatorname{Ag}(5)-\mathrm{O}(11)$ & 2.61 & & & & \\
\hline $\mathrm{O}(10)-\mathrm{W}(1)-\mathrm{O}(30)$ & $88.7(12)$ & $\mathrm{O}(10)-\mathrm{W}(1)-\mathrm{O}(31)$ & $87.7(12)$ & $\mathrm{O}(10)-\mathrm{W}(1)-\mathrm{O}(32)$ & $159.1(12)$ \\
\hline $\mathrm{O}(10)-\mathrm{W}(1)-\mathrm{O}(39)$ & $67.0(11)$ & $\mathrm{O}(30)-\mathrm{W}(1)-\mathrm{O}(31)$ & $157.0(12)$ & $\mathrm{O}(30)-\mathrm{W}(1)-\mathrm{O}(32)$ & $86.4(12)$ \\
\hline $\mathrm{O}(30)-\mathrm{W}(1)-\mathrm{O}(39)$ & $65.4(11)$ & $\mathrm{O}(31)-\mathrm{W}(1)-\mathrm{O}(32)$ & $89.0(12)$ & $\mathrm{O}(31)-\mathrm{W}(1)-\mathrm{O}(39)$ & $92.4(10)$ \\
\hline $\mathrm{O}(32)-\mathrm{W}(1)-\mathrm{O}(39)$ & $92.6(11)$ & $\mathrm{O}(33)-\mathrm{W}(1)-\mathrm{O}(10)$ & $100.3(13)$ & $\mathrm{O}(33)-\mathrm{W}(1)-\mathrm{O}(30)$ & $104.4(12)$ \\
\hline $\mathrm{O}(33)-\mathrm{W}(1)-\mathrm{O}(31)$ & $98.6(12)$ & $\mathrm{O}(33)-\mathrm{W}(1)-\mathrm{O}(32)$ & $100.6(13)$ & $\mathrm{O}(33)-\mathrm{W}(1)-\mathrm{O}(39)$ & $162.9(12)$ \\
\hline $\mathrm{O}(3)-\mathrm{W}(2)-\mathrm{O}(4)$ & $98.1(12)$ & $\mathrm{O}(3)-\mathrm{W}(2)-\mathrm{O}(22)$ & $98.1(12)$ & $\mathrm{O}(3)-\mathrm{W}(2)-\mathrm{O}(28)$ & $99.8(12)$ \\
\hline $\mathrm{O}(3)-\mathrm{W}(2)-\mathrm{O}(40)$ & $158.3(15)$ & $\mathrm{O}(3)-\mathrm{W}(2)-\mathrm{O}(41)$ & $159.4(11)$ & $\mathrm{O}(3)-\mathrm{W}(2)-\mathrm{O}(0 \mathrm{AA})$ & $99.6(12)$ \\
\hline $\mathrm{O}(4)-\mathrm{W}(2)-\mathrm{O}(22)$ & $85.4(11)$ & $\mathrm{O}(4)-\mathrm{W}(2)-\mathrm{O}(40)$ & $63.8(14)$ & $\mathrm{O}(4)-\mathrm{W}(2)-\mathrm{O}(41)$ & $94.3(10)$ \\
\hline $\mathrm{O}(4)-\mathrm{W}(2)-\mathrm{O}(0 \mathrm{AA})$ & $162.0(12)$ & $\mathrm{O}(22)-\mathrm{W}(2)-\mathrm{O}(40)$ & $92.6(15)$ & $\mathrm{O}(22)-\mathrm{W}(2)-\mathrm{O}(41)$ & $66.6(10)$ \\
\hline $\mathrm{O}(22)-\mathrm{W}(2)-\mathrm{O}(0 \mathrm{AA})$ & $89.3(11)$ & $\mathrm{O}(28)-\mathrm{W}(2)-\mathrm{O}(4)$ & $88.8(11)$ & $\mathrm{O}(28)-\mathrm{W}(2)-\mathrm{O}(22)$ & $161.8(11)$ \\
\hline $\mathrm{O}(28)-\mathrm{W}(2)-\mathrm{O}(40)$ & $69.4(15)$ & $\mathrm{O}(28)-\mathrm{W}(2)-\mathrm{O}(41)$ & $96.7(10)$ & $\mathrm{O}(28)-\mathrm{W}(2)-\mathrm{O}(0 \mathrm{AA})$ & $90.8(11)$ \\
\hline $\mathrm{O}(8)-\mathrm{W}(3)-\mathrm{O}(15)$ & $99.5(12)$ & $\mathrm{O}(8)-\mathrm{W}(3)-\mathrm{O}(16)$ & $99.0(12)$ & $\mathrm{O}(8)-\mathrm{W}(3)-\mathrm{O}(26)$ & $101.1(12)$ \\
\hline $\mathrm{O}(8)-\mathrm{W}(3)-\mathrm{O}(30)$ & $100.5(12)$ & $\mathrm{O}(8)-\mathrm{W}(3)-\mathrm{O}(38)$ & $159.5(15)$ & $\mathrm{O}(8)-\mathrm{W}(3)-\mathrm{O}(39)$ & $156.4(11)$ \\
\hline $\mathrm{O}(15)-\mathrm{W}(3)-\mathrm{O}(38)$ & $64.8(15)$ & $\mathrm{O}(15)-\mathrm{W}(3)-\mathrm{O}(39)$ & $97.4(10)$ & $\mathrm{O}(16)-\mathrm{W}(3)-\mathrm{O}(15)$ & $88.0(12)$ \\
\hline $\mathrm{O}(16)-\mathrm{W}(3)-\mathrm{O}(38)$ & $93.7(16)$ & $\mathrm{O}(16)-\mathrm{W}(3)-\mathrm{O}(39)$ & $65.2(10)$ & $\mathrm{O}(26)-\mathrm{W}(3)-\mathrm{O}(15)$ & $88.4(12)$ \\
\hline $\mathrm{O}(26)-\mathrm{W}(3)-\mathrm{O}(16)$ & $160.0(12)$ & $\mathrm{O}(26)-\mathrm{W}(3)-\mathrm{O}(30)$ & $89.1(12)$ & $\mathrm{O}(26)-\mathrm{W}(3)-\mathrm{O}(38)$ & $67.0(16)$ \\
\hline $\mathrm{O}(26)-\mathrm{W}(3)-\mathrm{O}(39)$ & $95.7(11)$ & $\mathrm{O}(30)-\mathrm{W}(3)-\mathrm{O}(15)$ & $160.0(12)$ & $\mathrm{O}(30)-\mathrm{W}(3)-\mathrm{O}(16)$ & $87.4(11)$ \\
\hline $\mathrm{O}(30)-\mathrm{W}(3)-\mathrm{O}(38)$ & $96.1(15)$ & $\mathrm{O}(30)-\mathrm{W}(3)-\mathrm{O}(39)$ & $63.1(10)$ & $\mathrm{O}(38)-\mathrm{W}(3)-\mathrm{O}(39)$ & $44.0(14)$ \\
\hline $\mathrm{O}(1)-\mathrm{W}(4)-\mathrm{O}(2)$ & $101.3(12)$ & $\mathrm{O}(1)-\mathrm{W}(4)-\mathrm{O}(4)$ & $99.4(11)$ & $\mathrm{O}(1)-\mathrm{W}(4)-\mathrm{O}(12)$ & $98.0(12)$ \\
\hline $\mathrm{O}(1)-\mathrm{W}(4)-\mathrm{O}(24)$ & $103.0(12)$ & $\mathrm{O}(1)-\mathrm{W}(4)-\mathrm{O}(36)$ & $162.6(16)$ & $\mathrm{O}(1)-\mathrm{W}(4)-\mathrm{O}(40)$ & $157.4(16)$ \\
\hline $\mathrm{O}(2)-\mathrm{W}(4)-\mathrm{O}(4)$ & $88.1(11)$ & $\mathrm{O}(2)-\mathrm{W}(4)-\mathrm{O}(12)$ & $160.6(11)$ & $\mathrm{O}(2)-\mathrm{W}(4)-\mathrm{O}(36)$ & $66.8(14)$ \\
\hline $\mathrm{O}(2)-\mathrm{W}(4)-\mathrm{O}(40)$ & $94.9(15)$ & $\mathrm{O}(4)-\mathrm{W}(4)-\mathrm{O}(12)$ & $86.7(11)$ & $\mathrm{O}(4)-\mathrm{W}(4)-\mathrm{O}(36)$ & $93.0(15)$ \\
\hline $\mathrm{O}(4)-\mathrm{W}(4)-\mathrm{O}(40)$ & $65.3(14)$ & $\mathrm{O}(12)-\mathrm{W}(4)-\mathrm{O}(36)$ & $94.9(15)$ & $\mathrm{O}(12)-\mathrm{W}(4)-\mathrm{O}(40)$ & $66.1(15)$ \\
\hline $\mathrm{O}(24)-\mathrm{W}(4)-\mathrm{O}(2)$ & $91.7(11)$ & $\mathrm{O}(24)-\mathrm{W}(4)-\mathrm{O}(4)$ & $157.2(11)$ & $\mathrm{O}(24)-\mathrm{W}(4)-\mathrm{O}(12)$ & $86.0(11)$ \\
\hline $\mathrm{O}(24)-\mathrm{W}(4)-\mathrm{O}(36)$ & $66.1(15)$ & $\mathrm{O}(24)-\mathrm{W}(4)-\mathrm{O}(40)$ & $92.0(15)$ & $\mathrm{O}(40)-\mathrm{W}(4)-\mathrm{O}(36)$ & $40.0(18)$ \\
\hline $\mathrm{O}(13)-\mathrm{W}(5)-\mathrm{O}(23)$ & $96.7(12)$ & $\mathrm{O}(13)-\mathrm{W}(5)-\mathrm{O}(25)$ & $101.1(12)$ & $\mathrm{O}(13)-\mathrm{W}(5)-\mathrm{O}(32)$ & $103.2(12)$ \\
\hline $\mathrm{O}(13)-\mathrm{W}(5)-\mathrm{O}(34)$ & $99.3(13)$ & $\mathrm{O}(13)-\mathrm{W}(5)-\mathrm{O}(37)$ & $154.8(16)$ & $\mathrm{O}(23)-\mathrm{W}(5)-\mathrm{O}(37)$ & $63.1(15)$ \\
\hline $\mathrm{O}(25)-\mathrm{W}(5)-\mathrm{O}(23)$ & $86.7(11)$ & $\mathrm{O}(25)-\mathrm{W}(5)-\mathrm{O}(37)$ & $93.1(15)$ & $\mathrm{O}(32)-\mathrm{W}(5)-\mathrm{O}(23)$ & $160.1(12)$ \\
\hline $\mathrm{O}(32)-\mathrm{W}(5)-\mathrm{O}(25)$ & $90.2(12)$ & $\mathrm{O}(32)-\mathrm{W}(5)-\mathrm{O}(34)$ & $87.8(12)$ & $\mathrm{O}(32)-\mathrm{W}(5)-\mathrm{O}(37)$ & $97.4(16)$ \\
\hline $\mathrm{O}(34)-\mathrm{W}(5)-\mathrm{O}(23)$ & $88.2(11)$ & $\mathrm{O}(34)-\mathrm{W}(5)-\mathrm{O}(25)$ & $159.5(12)$ & $\mathrm{O}(34)-\mathrm{W}(5)-\mathrm{O}(37)$ & $67.0(15)$ \\
\hline $\mathrm{O}(12)-\mathrm{W}(6)-\mathrm{O}(27)$ & $161.7(12)$ & $\mathrm{O}(12)-\mathrm{W}(6)-\mathrm{O}(28)$ & $88.7(11)$ & $\mathrm{O}(12)-\mathrm{W}(6)-\mathrm{O}(29)$ & $88.3(11)$ \\
\hline $\mathrm{O}(12)-\mathrm{W}(6)-\mathrm{O}(40)$ & $65.4(15)$ & $\mathrm{O}(14)-\mathrm{W}(6)-\mathrm{O}(12)$ & $100.4(12)$ & $\mathrm{O}(14)-\mathrm{W}(6)-\mathrm{O}(27)$ & $97.8(13)$ \\
\hline $\mathrm{O}(14)-\mathrm{W}(6)-\mathrm{O}(28)$ & $98.9(12)$ & $\mathrm{O}(14)-\mathrm{W}(6)-\mathrm{O}(29)$ & $100.0(12)$ & $\mathrm{O}(14)-\mathrm{W}(6)-\mathrm{O}(40)$ & $160.1(15)$ \\
\hline
\end{tabular}




\begin{tabular}{|c|c|c|c|c|c|}
\hline $\mathrm{O}(27)-\mathrm{W}(6)-\mathrm{O}(28)$ & $86.6(11)$ & $\mathrm{O}(27)-\mathrm{W}(6)-\mathrm{O}(40)$ & $96.5(15)$ & $\mathrm{O}(28)-\mathrm{W}(6)-\mathrm{O}(40)$ & $68.1(14)$ \\
\hline $\mathrm{O}(29)-\mathrm{W}(6)-\mathrm{O}(27)$ & $90.4(11)$ & $\mathrm{O}(29)-\mathrm{W}(6)-\mathrm{O}(28)$ & $161.0(12)$ & $\mathrm{O}(29)-\mathrm{W}(6)-\mathrm{O}(40)$ & $93.6(15)$ \\
\hline $\mathrm{O}(7)-\mathrm{W}(7)-\mathrm{O}(17)$ & $99.1(12)$ & $\mathrm{O}(7)-\mathrm{W}(7)-\mathrm{O}(21)$ & $101.2(12)$ & $\mathrm{O}(7)-\mathrm{W}(7)-\mathrm{O}(24)$ & $102.1(12)$ \\
\hline $\mathrm{O}(7)-\mathrm{W}(7)-\mathrm{O}(36)$ & $156.6(14)$ & $\mathrm{O}(7)-\mathrm{W}(7)-\mathrm{O}(41)$ & $161.6(10)$ & $\mathrm{O}(7)-\mathrm{W}(7)-\mathrm{O}(0 \mathrm{AA})$ & $97.9(12)$ \\
\hline $\mathrm{O}(17)-\mathrm{W}(7)-\mathrm{O}(24)$ & $88.2(11)$ & $\mathrm{O}(17)-\mathrm{W}(7)-\mathrm{O}(36)$ & $64.3(14)$ & $\mathrm{O}(17)-\mathrm{W}(7)-\mathrm{O}(41)$ & 93.3(10) \\
\hline $\mathrm{O}(21)-\mathrm{W}(7)-\mathrm{O}(17)$ & $159.6(11)$ & $\mathrm{O}(21)-\mathrm{W}(7)-\mathrm{O}(24)$ & $88.2(11)$ & $\mathrm{O}(21)-\mathrm{W}(7)-\mathrm{O}(36)$ & $96.3(14)$ \\
\hline $\mathrm{O}(21)-\mathrm{W}(7)-\mathrm{O}(41)$ & $66.8(10)$ & $\mathrm{O}(24)-\mathrm{W}(7)-\mathrm{O}(36)$ & $62.9(15)$ & $\mathrm{O}(24)-\mathrm{W}(7)-\mathrm{O}(41)$ & $91.7(10)$ \\
\hline $\mathrm{O}(41)-\mathrm{W}(7)-\mathrm{O}(36)$ & $41.8(13)$ & $\mathrm{O}(0 \mathrm{AA})-\mathrm{W}(7)-\mathrm{O}(17)$ & $88.6(11)$ & $\mathrm{O}(0 \mathrm{AA})-\mathrm{W}(7)-\mathrm{O}(21)$ & $88.0(11)$ \\
\hline $\mathrm{O}(0 \mathrm{AA})-\mathrm{W}(7)-\mathrm{O}(24)$ & $160.0(12)$ & $\mathrm{O}(0 \mathrm{AA})-\mathrm{W}(7)-\mathrm{O}(36)$ & $98.0(14)$ & $\mathrm{O}(0 \mathrm{AA})-\mathrm{W}(7)-\mathrm{O}(41)$ & $68.8(10)$ \\
\hline $\mathrm{O}(6)-\mathrm{W}(8)-\mathrm{O}(10)$ & $88.4(11)$ & $\mathrm{O}(6)-\mathrm{W}(8)-\mathrm{O}(37)$ & $68.1(16)$ & $\mathrm{O}(6)-\mathrm{W}(8)-\mathrm{O}(39)$ & $94.6(11)$ \\
\hline $\mathrm{O}(10)-\mathrm{W}(8)-\mathrm{O}(37)$ & $98.1(16)$ & $\mathrm{O}(10)-\mathrm{W}(8)-\mathrm{O}(39)$ & $64.9(10)$ & $\mathrm{O}(11)-\mathrm{W}(8)-\mathrm{O}(6)$ & $98.0(12)$ \\
\hline $\mathrm{O}(11)-\mathrm{W}(8)-\mathrm{O}(10)$ & $97.6(12)$ & $\mathrm{O}(11)-\mathrm{W}(8)-\mathrm{O}(16)$ & $99.9(13)$ & $\mathrm{O}(11)-\mathrm{W}(8)-\mathrm{O}(23)$ & $99.7(12)$ \\
\hline $\mathrm{O}(11)-\mathrm{W}(8)-\mathrm{O}(37)$ & $158.7(16)$ & $\mathrm{O}(11)-\mathrm{W}(8)-\mathrm{O}(39)$ & $158.1(11)$ & $\mathrm{O}(16)-\mathrm{W}(8)-\mathrm{O}(6)$ & $162.0(13)$ \\
\hline $\mathrm{O}(16)-\mathrm{W}(8)-\mathrm{O}(10)$ & $87.8(11)$ & $\mathrm{O}(16)-\mathrm{W}(8)-\mathrm{O}(23)$ & $90.8(12)$ & $\mathrm{O}(16)-\mathrm{W}(8)-\mathrm{O}(37)$ & $95.1(16)$ \\
\hline $\mathrm{O}(16)-\mathrm{W}(8)-\mathrm{O}(39)$ & $67.9(11)$ & $\mathrm{O}(23)-\mathrm{W}(8)-\mathrm{O}(6)$ & $87.7(11)$ & $\mathrm{O}(23)-\mathrm{W}(8)-\mathrm{O}(10)$ & $162.7(12)$ \\
\hline $\mathrm{O}(23)-\mathrm{W}(8)-\mathrm{O}(37)$ & $64.8(16)$ & $\mathrm{O}(23)-\mathrm{W}(8)-\mathrm{O}(39)$ & $98.7(11)$ & $\mathrm{O}(37)-\mathrm{W}(8)-\mathrm{O}(39)$ & $43.2(15)$ \\
\hline $\mathrm{O}(9)-\mathrm{W}(9)-\mathrm{O}(15)$ & $100.9(12)$ & $\mathrm{O}(9)-\mathrm{W}(9)-\mathrm{O}(25)$ & $100.3(12)$ & $\mathrm{O}(9)-\mathrm{W}(9)-\mathrm{O}(31)$ & $98.1(11)$ \\
\hline $\mathrm{O}(9)-\mathrm{W}(9)-\mathrm{O}(35)$ & $101.1(12)$ & $\mathrm{O}(9)-\mathrm{W}(9)-\mathrm{O}(38)$ & $160.1(16)$ & $\mathrm{O}(15)-\mathrm{W}(9)-\mathrm{O}(31)$ & $160.6(11)$ \\
\hline $\mathrm{O}(15)-\mathrm{W}(9)-\mathrm{O}(38)$ & $66.1(15)$ & $\mathrm{O}(25)-\mathrm{W}(9)-\mathrm{O}(15)$ & $87.7(12)$ & $\mathrm{O}(25)-\mathrm{W}(9)-\mathrm{O}(31)$ & $92.3(12)$ \\
\hline $\mathrm{O}(25)-\mathrm{W}(9)-\mathrm{O}(35)$ & $158.6(11)$ & $\mathrm{O}(25)-\mathrm{W}(9)-\mathrm{O}(38)$ & $94.4(16)$ & $\mathrm{O}(31)-\mathrm{W}(9)-\mathrm{O}(38)$ & $94.5(15)$ \\
\hline $\mathrm{O}(35)-\mathrm{W}(9)-\mathrm{O}(15)$ & $89.5(12)$ & $\mathrm{O}(35)-\mathrm{W}(9)-\mathrm{O}(31)$ & $83.4(12)$ & $\mathrm{O}(35)-\mathrm{W}(9)-\mathrm{O}(38)$ & $65.2(16)$ \\
\hline $\mathrm{O}(5)-\mathrm{W}(10)-\mathrm{O}(6)$ & $97.5(12)$ & $\mathrm{O}(5)-\mathrm{W}(10)-\mathrm{O}(26)$ & $100.2(12)$ & $\mathrm{O}(5)-\mathrm{W}(10)-\mathrm{O}(34)$ & $98.8(12)$ \\
\hline $\mathrm{O}(5)-\mathrm{W}(10)-\mathrm{O}(35)$ & $102.8(12)$ & $\mathrm{O}(5)-\mathrm{W}(10)-\mathrm{O}(37)$ & $154.5(16)$ & $\mathrm{O}(5)-\mathrm{W}(10)-\mathrm{O}(38)$ & $159.0(15)$ \\
\hline $\mathrm{O}(6)-\mathrm{W}(10)-\mathrm{O}(26)$ & $162.2(12)$ & $\mathrm{O}(6)-\mathrm{W}(10)-\mathrm{O}(34)$ & $90.1(11)$ & $\mathrm{O}(6)-\mathrm{W}(10)-\mathrm{O}(37)$ & $65.4(15)$ \\
\hline $\mathrm{O}(6)-\mathrm{W}(10)-\mathrm{O}(38)$ & $98.0(15)$ & $\mathrm{O}(26)-\mathrm{W}(10)-\mathrm{O}(37)$ & $97.4(15)$ & $\mathrm{O}(26)-\mathrm{W}(10)-\mathrm{O}(38)$ & $65.3(15)$ \\
\hline $\mathrm{O}(34)-\mathrm{W}(10)-\mathrm{O}(26)$ & $85.8(12)$ & $\mathrm{O}(34)-\mathrm{W}(10)-\mathrm{O}(37)$ & $64.2(15)$ & $\mathrm{O}(34)-\mathrm{W}(10)-\mathrm{O}(38)$ & $95.3(15)$ \\
\hline $\mathrm{O}(35)-\mathrm{W}(10)-\mathrm{O}(6)$ & $87.8(12)$ & $\mathrm{O}(35)-\mathrm{W}(10)-\mathrm{O}(26)$ & $89.8(12)$ & $\mathrm{O}(35)-\mathrm{W}(10)-\mathrm{O}(34)$ & $158.3(12)$ \\
\hline $\mathrm{O}(35)-\mathrm{W}(10)-\mathrm{O}(37)$ & $95.5(15)$ & $\mathrm{O}(35)-\mathrm{W}(10)-\mathrm{O}(38)$ & $63.7(15)$ & $\mathrm{O}(38)-\mathrm{W}(10)-\mathrm{O}(38)$ & $46.5(17)$ \\
\hline $\mathrm{O}(2)-\mathrm{W}(11)-\mathrm{O}(36)$ & $66.4(15)$ & $\mathrm{O}(17)-\mathrm{W}(11)-\mathrm{O}(2)$ & $89.1(11)$ & $\mathrm{O}(17)-\mathrm{W}(11)-\mathrm{O}(19)$ & $161.6(12)$ \\
\hline $\mathrm{O}(17)-\mathrm{W}(11)-\mathrm{O}(36)$ & $67.4(15)$ & $\mathrm{O}(18)-\mathrm{W}(11)-\mathrm{O}(2)$ & $96.0(12)$ & $\mathrm{O}(18)-\mathrm{W}(11)-\mathrm{O}(17)$ & $100.3(12)$ \\
\hline $\mathrm{O}(18)-\mathrm{W}(11)-\mathrm{O}(19)$ & $98.0(12)$ & $\mathrm{O}(18)-\mathrm{W}(11)-\mathrm{O}(27)$ & $102.2(13)$ & $\mathrm{O}(18)-\mathrm{W}(11)-\mathrm{O}(36)$ & $157.8(16)$ \\
\hline $\mathrm{O}(19)-\mathrm{W}(11)-\mathrm{O}(2)$ & $87.0(11)$ & $\mathrm{O}(19)-\mathrm{W}(11)-\mathrm{O}(36)$ & $94.6(15)$ & $\mathrm{O}(27)-\mathrm{W}(11)-\mathrm{O}(2)$ & $161.7(12)$ \\
\hline $\mathrm{O}(27)-\mathrm{W}(11)-\mathrm{O}(17)$ & $89.4(11)$ & $\mathrm{O}(27)-\mathrm{W}(11)-\mathrm{O}(19)$ & $88.8(11)$ & $\mathrm{O}(27)-\mathrm{W}(11)-\mathrm{O}(36)$ & $96.3(15)$ \\
\hline $\mathrm{O}(19)-\mathrm{W}(12)-\mathrm{O}(41)$ & $96.9(10)$ & $\mathrm{O}(20)-\mathrm{W}(12)-\mathrm{O}(19)$ & $98.6(11)$ & $\mathrm{O}(20)-\mathrm{W}(12)-\mathrm{O}(21)$ & $99.6(11)$ \\
\hline $\mathrm{O}(20)-\mathrm{W}(12)-\mathrm{O}(22)$ & $100.1(11)$ & $\mathrm{O}(20)-\mathrm{W}(12)-\mathrm{O}(29)$ & $101.8(11)$ & $\mathrm{O}(20)-\mathrm{W}(12)-\mathrm{O}(41)$ & $159.0(11)$ \\
\hline $\mathrm{O}(21)-\mathrm{W}(12)-\mathrm{O}(19)$ & $161.7(11)$ & $\mathrm{O}(21)-\mathrm{W}(12)-\mathrm{O}(41)$ & $65.9(10)$ & $\mathrm{O}(22)-\mathrm{W}(12)-\mathrm{O}(19)$ & $89.8(12)$ \\
\hline $\mathrm{O}(22)-\mathrm{W}(12)-\mathrm{O}(21)$ & $88.7(11)$ & $\mathrm{O}(22)-\mathrm{W}(12)-\mathrm{O}(29)$ & $158.1(11)$ & $\mathrm{O}(22)-\mathrm{W}(12)-\mathrm{O}(41)$ & $65.9(10)$ \\
\hline $\mathrm{O}(29)-\mathrm{W}(12)-\mathrm{O}(19)$ & $88.0(11)$ & $\mathrm{O}(29)-\mathrm{W}(12)-\mathrm{O}(21)$ & $86.6(11)$ & $\mathrm{O}(29)-\mathrm{W}(12)-\mathrm{O}(41)$ & $92.7(10)$ \\
\hline $\mathrm{N}(3)-\operatorname{Ag}(1)-\mathrm{N}(10)$ & $95.7(12)$ & $\mathrm{N}(3)-\operatorname{Ag}(1)-\mathrm{N}(14)$ & $83.1(16)$ & $\mathrm{N}(9)-\operatorname{Ag}(1)-\mathrm{N}(3)$ & $167.0(11)$ \\
\hline $\mathrm{N}(9)-\operatorname{Ag}(1)-\mathrm{N}(10)$ & $97.2(12)$ & $\mathrm{N}(9)-\operatorname{Ag}(1)-\mathrm{N}(14)$ & $83.9(16)$ & $\mathrm{N}(10)-\operatorname{Ag}(1)-\mathrm{N}(14)$ & $178.3(16)$ \\
\hline $\mathrm{N}(11)-\operatorname{Ag}(2)-\mathrm{N}(13)$ & $119.1(10)$ & $\mathrm{N}(11)-\operatorname{Ag}(2)-\mathrm{N}(12)$ & $120.3(10)$ & $\mathrm{N}(13)-\operatorname{Ag}(2)-\mathrm{N}(12)$ & $120.5(9)$ \\
\hline $\mathrm{N}(2)-\operatorname{Ag}(3)-\mathrm{N}(6)$ & $107.9(16)$ & $\mathrm{N}(7)-\mathrm{Ag}(3)-\mathrm{N}(2)$ & $144.1(15)$ & $\mathrm{N}(7)-\operatorname{Ag}(3)-\mathrm{N}(6)$ & $108.0(16)$ \\
\hline$N(1)-\operatorname{Ag}(4)-N(21)$ & $114.3(13)$ & $\mathrm{N}(5)-\mathrm{Ag}(5)-\mathrm{N}(4)$ & $112.4(12)$ & $\mathrm{N}(3)-\mathrm{Ag}(1)-\mathrm{O}(5)$ & 92.40 \\
\hline $\mathrm{N}(3)-\mathrm{Ag}(1)-\mathrm{O}(20)$ & 91.75 & $\mathrm{~N}(9)-\operatorname{Ag}(1)-\mathrm{O}(5)$ & 91.56 & $\mathrm{~N}(9)-\operatorname{Ag}(1)-\mathrm{O}(20)$ & 90.93 \\
\hline $\mathrm{N}(10)-\operatorname{Ag}(1)-\mathrm{O}(5)$ & 75.84 & $\mathrm{~N}(10)-\mathrm{Ag}(1)-\mathrm{O}(20)$ & 74.79 & $\mathrm{~N}(14)-\mathrm{Ag}(1)-\mathrm{O}(5)$ & 105.42 \\
\hline
\end{tabular}




\begin{tabular}{|l|l|l|l|l|l|}
\hline $\mathrm{N}(14)-\operatorname{Ag}(1)-\mathrm{O}(20)$ & 103.95 & $\mathrm{~N}(2)-\operatorname{Ag}(3)-\mathrm{O}(13)$ & 86.149 & $\mathrm{~N}(2)-\operatorname{Ag}(3)-\mathrm{O}(14)$ & 92.378 \\
\hline $\mathrm{N}(6)-\operatorname{Ag}(3)-\mathrm{O}(13)$ & 93.613 & $\mathrm{~N}(6)-\operatorname{Ag}(3)-\mathrm{O}(14)$ & 91.684 & $\mathrm{~N}(7)-\operatorname{Ag}(3)-\mathrm{O}(13)$ & 91.082 \\
\hline $\mathrm{N}(7)-\operatorname{Ag}(3)-\mathrm{O}(14)$ & 87.108 & $\mathrm{~N}(1)-\operatorname{Ag}(4)-\mathrm{O}(9)$ & $96.8(11)$ & $\mathrm{N}(1)-\operatorname{Ag}(4)-\mathrm{O}(18)$ & $124.2(10)$ \\
\hline $\mathrm{N}(21)-\operatorname{Ag}(4)-\mathrm{O}(9)$ & $112.8(10)$ & $\mathrm{N}(21)-\operatorname{Ag}(4)-\mathrm{O}(18)$ & $86.4(10)$ & $\mathrm{N}(4)-\operatorname{Ag}(5)-\mathrm{O}(3)$ & $111.0(10)$ \\
\hline $\mathrm{N}(4)-\operatorname{Ag}(5)-\mathrm{O}(11)$ & 88.782 & $\mathrm{~N}(5)-\operatorname{Ag}(5)-\mathrm{O}(3)$ & $96.1(11)$ & $\mathrm{N}(5)-\operatorname{Ag}(5)-\mathrm{O}(11)$ & 124.587 \\
\hline $\mathrm{O}(5)-\operatorname{Ag}(1)-\mathrm{O}(20)$ & 150.61 & $\mathrm{O}(13)-\operatorname{Ag}(3)-\mathrm{O}(14)$ & 174.702 & $\mathrm{O}(9)-\operatorname{Ag}(4)-\mathrm{O}(18)$ & $123.1(9)$ \\
\hline $\mathrm{O}(3)-\operatorname{Ag}(5)-\mathrm{O}(11)$ & 124.176 & & & & \\
\hline
\end{tabular}

Symmetry transformations used to generate equivalent atoms: \#1 -x,-y,-z; \#2 -x+1/2,-y+1/2,-z

Table S3 Selected bond lengths $(\AA)$ and bond angles $\left({ }^{\circ}\right)$ of compound 2

\begin{tabular}{|c|c|c|c|c|c|}
\hline $\mathrm{Si}(1)-\mathrm{O}(2)$ & $1.55(2)$ & $\mathrm{Si}(1)-\mathrm{O}(20)$ & $1.64(3)$ & $\mathrm{Si}(1)-\mathrm{O}(21)$ & $1.65(3)$ \\
\hline $\mathrm{Si}(1)-\mathrm{O}(0 \mathrm{AA})$ & $1.59(2)$ & $\mathrm{W}(1)-\mathrm{O}(2)$ & $2.43(2)$ & $\mathrm{W}(1)-\mathrm{O}(3)$ & $1.883(17)$ \\
\hline $\mathrm{W}(1)-\mathrm{O}(4)$ & $1.647(15)$ & $\mathrm{W}(1)-\mathrm{O}(10)$ & $1.894(19)$ & $\mathrm{W}(1)-\mathrm{O}(15)$ & $1.893(17)$ \\
\hline $\mathrm{W}(1)-\mathrm{O}(18)$ & $1.861(17)$ & $\mathrm{W}(1)-\mathrm{O}(21)$ & $2.32(3)$ & $\mathrm{W}(2)-\mathrm{O}(1)$ & $1.667(15)$ \\
\hline $\mathrm{W}(2)-\mathrm{O}(2)$ & $2.46(2)$ & $\mathrm{W}(2)-\mathrm{O}(3)$ & $1.884(16)$ & $\mathrm{W}(2)-\mathrm{O}(5)$ & $1.899(17)$ \\
\hline $\mathrm{W}(2)-\mathrm{O}(7)$ & $1.892(18)$ & $\mathrm{W}(2)-\mathrm{O}(11)$ & $1.884(19)$ & $\mathrm{W}(2)-\mathrm{O}(20)$ & $2.34(3)$ \\
\hline $\mathrm{W}(3)-\mathrm{O}(2)$ & $2.38(2)$ & $\mathrm{W}(3)-\mathrm{O}(5)$ & $1.890(17)$ & $\mathrm{W}(3)-\mathrm{O}(14)$ & $1.855(16)$ \\
\hline $\mathrm{W}(3)-\mathrm{O}(16)$ & $1.654(15)$ & $\mathrm{W}(3)-\mathrm{O}(17)$ & $1.867(15)$ & $\mathrm{W}(3)-\mathrm{O}(18)$ & $1.898(16)$ \\
\hline $\mathrm{W}(3)-\mathrm{O}(0 \mathrm{AA})$ & $2.32(2)$ & $\mathrm{W}(4)-\mathrm{O}(8)$ & $1.670(14)$ & $\mathrm{W}(4)-\mathrm{O}(9)$ & $1.890(19)$ \\
\hline $\mathrm{W}(4)-\mathrm{O}(10)$ & $1.874(19)$ & $\mathrm{W}(4)-\mathrm{O}(17)$ & $1.912(17)$ & $\mathrm{W}(4)-\mathrm{O}(19)$ & $1.920(17)$ \\
\hline $\mathrm{W}(4)-\mathrm{O}(21)$ & $2.34(3)$ & $\mathrm{W}(4)-\mathrm{O}(0 \mathrm{AA})$ & $2.40(2)$ & $\mathrm{W}(5)-\mathrm{O}(9)$ & $1.89(2)$ \\
\hline $\mathrm{W}(5)-\mathrm{O}(11)$ & $1.85(2)$ & $\mathrm{W}(5)-\mathrm{O}(12)$ & $1.685(14)$ & $\mathrm{W}(5)-\mathrm{O}(13)$ & $1.848(19)$ \\
\hline $\mathrm{W}(5)-\mathrm{O}(15)$ & $1.886(17)$ & $\mathrm{W}(5)-\mathrm{O}(20)$ & $2.42(3)$ & $\mathrm{W}(5)-\mathrm{O}(21)$ & $2.44(3)$ \\
\hline $\mathrm{W}(6)-\mathrm{O}(6)$ & $1.675(14)$ & $\mathrm{W}(6)-\mathrm{O}(7)$ & $1.908(19)$ & $\mathrm{W}(6)-\mathrm{O}(13)$ & $1.910(18)$ \\
\hline $\mathrm{W}(6)-\mathrm{O}(14)$ & $1.902(18)$ & $\mathrm{W}(6)-\mathrm{O}(19)$ & $1.852(17)$ & $\mathrm{W}(6)-\mathrm{O}(20)$ & $2.36(3)$ \\
\hline $\mathrm{W}(6)-\mathrm{O}(0 \mathrm{AA})$ & $2.49(2)$ & $\operatorname{Ag}(1)-\mathrm{N}(4)$ & $2.25(3)$ & $\operatorname{Ag}(1)-\mathrm{N}(6)$ & $2.26(6)$ \\
\hline $\operatorname{Ag}(2)-N(1)$ & $2.25(3)$ & $\operatorname{Ag}(2)-\mathrm{N}(2)$ & $2.252(17)$ & $\operatorname{Ag}(3)-N(3)$ & $2.34(3)$ \\
\hline $\operatorname{Ag}(3)-N(7)$ & $2.25(5)$ & $\operatorname{Ag}(3)-\mathrm{N}(8)$ & $2.30(4)$ & $\operatorname{Ag}(4)-\mathrm{N}(5)$ & $2.18(3)$ \\
\hline $\operatorname{Ag}(4)-N(9)$ & $2.244(17)$ & $\mathrm{Ag}(1)-\mathrm{O}(4)$ & 2.692 & $\operatorname{Ag}(4)-O(1)$ & $2.524(17)$ \\
\hline $\mathrm{Ag}(4)-\mathrm{O}(8)$ & 2.623 & $\mathrm{Ag}(4)-\mathrm{O}(22)$ & $1.195(16)$ & & \\
\hline $\mathrm{O}(3)-\mathrm{W}(1)-\mathrm{O}(2)$ & $65.6(8)$ & $\mathrm{O}(3)-\mathrm{W}(1)-\mathrm{O}(10)$ & $86.8(8)$ & $\mathrm{O}(3)-\mathrm{W}(1)-\mathrm{O}(15)$ & $158.4(8)$ \\
\hline $\mathrm{O}(3)-\mathrm{W}(1)-\mathrm{O}(21)$ & $94.1(9)$ & $\mathrm{O}(4)-\mathrm{W}(1)-\mathrm{O}(2)$ & $157.0(9)$ & $\mathrm{O}(4)-\mathrm{W}(1)-\mathrm{O}(3)$ & $99.4(9)$ \\
\hline $\mathrm{O}(4)-\mathrm{W}(1)-\mathrm{O}(10)$ & $101.0(9)$ & $\mathrm{O}(4)-\mathrm{W}(1)-\mathrm{O}(15)$ & $102.1(9)$ & $\mathrm{O}(4)-\mathrm{W}(1)-\mathrm{O}(18)$ & $101.4(9)$ \\
\hline $\mathrm{O}(4)-\mathrm{W}(1)-\mathrm{O}(21)$ & $157.9(9)$ & $\mathrm{O}(10)-\mathrm{W}(1)-\mathrm{O}(2)$ & $95.7(9)$ & $\mathrm{O}(10)-\mathrm{W}(1)-\mathrm{O}(21)$ & $62.2(9)$ \\
\hline $\mathrm{O}(15)-\mathrm{W}(1)-\mathrm{O}(2)$ & $94.2(9)$ & $\mathrm{O}(15)-\mathrm{W}(1)-\mathrm{O}(10)$ & $87.7(8)$ & $\mathrm{O}(15)-\mathrm{W}(1)-\mathrm{O}(21)$ & $65.1(8)$ \\
\hline $\mathrm{O}(18)-\mathrm{W}(1)-\mathrm{O}(2)$ & $62.7(8)$ & $\mathrm{O}(18)-\mathrm{W}(1)-\mathrm{O}(3)$ & $88.9(7)$ & $\mathrm{O}(18)-\mathrm{W}(1)-\mathrm{O}(10)$ & $157.6(9)$ \\
\hline $\mathrm{O}(18)-\mathrm{W}(1)-\mathrm{O}(15)$ & $88.2(8)$ & $\mathrm{O}(18)-\mathrm{W}(1)-\mathrm{O}(21)$ & $96.2(9)$ & $\mathrm{O}(21)-\mathrm{W}(1)-\mathrm{O}(2)$ & $45.0(8)$ \\
\hline $\mathrm{O}(1)-\mathrm{W}(2)-\mathrm{O}(2)$ & $158.0(8)$ & $\mathrm{O}(1)-\mathrm{W}(2)-\mathrm{O}(3)$ & $102.1(8)$ & $\mathrm{O}(1)-\mathrm{W}(2)-\mathrm{O}(5)$ & $100.3(8)$ \\
\hline $\mathrm{O}(1)-\mathrm{W}(2)-\mathrm{O}(7)$ & $100.0(9)$ & $\mathrm{O}(1)-\mathrm{W}(2)-\mathrm{O}(11)$ & $101.6(9)$ & $\mathrm{O}(1)-\mathrm{W}(2)-\mathrm{O}(20)$ & $156.6(9)$ \\
\hline $\mathrm{O}(3)-\mathrm{W}(2)-\mathrm{O}(2)$ & $65.0(8)$ & $\mathrm{O}(3)-\mathrm{W}(2)-\mathrm{O}(5)$ & $88.9(7)$ & $\mathrm{O}(3)-\mathrm{W}(2)-\mathrm{O}(7)$ & $87.8(8)$ \\
\hline $\mathrm{O}(3)-\mathrm{W}(2)-\mathrm{O}(11)$ & $156.3(9)$ & $\mathrm{O}(3)-\mathrm{W}(2)-\mathrm{O}(20)$ & $94.9(9)$ & $\mathrm{O}(5)-\mathrm{W}(2)-\mathrm{O}(2)$ & $63.3(8)$ \\
\hline $\mathrm{O}(5)-\mathrm{W}(2)-\mathrm{O}(20)$ & $96.0(9)$ & $\mathrm{O}(7)-\mathrm{W}(2)-\mathrm{O}(2)$ & $97.3(9)$ & $\mathrm{O}(7)-\mathrm{W}(2)-\mathrm{O}(5)$ & $159.6(9)$ \\
\hline $\mathrm{O}(7)-\mathrm{W}(2)-\mathrm{O}(20)$ & $64.3(9)$ & $\mathrm{O}(11)-\mathrm{W}(2)-\mathrm{O}(2)$ & $92.2(9)$ & $\mathrm{O}(11)-\mathrm{W}(2)-\mathrm{O}(5)$ & $86.2(8)$ \\
\hline $\mathrm{O}(11)-\mathrm{W}(2)-\mathrm{O}(7)$ & $88.7(8)$ & $\mathrm{O}(11)-\mathrm{W}(2)-\mathrm{O}(20)$ & $62.6(9)$ & $\mathrm{O}(20)-\mathrm{W}(2)-\mathrm{O}(2)$ & $45.3(9)$ \\
\hline
\end{tabular}




\begin{tabular}{|c|c|c|c|c|c|}
\hline $\mathrm{O}(5)-\mathrm{W}(3)-\mathrm{O}(2)$ & $65.1(8)$ & $\mathrm{O}(5)-\mathrm{W}(3)-\mathrm{O}(18)$ & $87.9(7)$ & $\mathrm{O}(5)-\mathrm{W}(3)-\mathrm{O}(0 \mathrm{AA})$ & $93.1(8)$ \\
\hline $\mathrm{O}(14)-\mathrm{W}(3)-\mathrm{O}(2)$ & $92.7(9)$ & $\mathrm{O}(14)-\mathrm{W}(3)-\mathrm{O}(5)$ & $157.2(9)$ & $\mathrm{O}(14)-\mathrm{W}(3)-\mathrm{O}(17)$ & $90.0(7)$ \\
\hline $\mathrm{O}(14)-\mathrm{W}(3)-\mathrm{O}(18)$ & $86.2(8)$ & $\mathrm{O}(14)-\mathrm{W}(3)-\mathrm{O}(0 \mathrm{AA})$ & $65.4(9)$ & $\mathrm{O}(16)-\mathrm{W}(3)-\mathrm{O}(2)$ & $156.3(10)$ \\
\hline $\mathrm{O}(16)-\mathrm{W}(3)-\mathrm{O}(5)$ & $99.9(10)$ & $\mathrm{O}(16)-\mathrm{W}(3)-\mathrm{O}(14)$ & $102.8(10)$ & $\mathrm{O}(16)-\mathrm{W}(3)-\mathrm{O}(17)$ & $103.6(10)$ \\
\hline $\mathrm{O}(16)-\mathrm{W}(3)-\mathrm{O}(18)$ & $99.5(10)$ & $\mathrm{O}(16)-\mathrm{W}(3)-\mathrm{O}(0 \mathrm{AA})$ & $161.6(10)$ & $\mathrm{O}(17)-\mathrm{W}(3)-\mathrm{O}(2)$ & 94.2(9) \\
\hline $\mathrm{O}(17)-\mathrm{W}(3)-\mathrm{O}(5)$ & $86.9(7)$ & $\mathrm{O}(17)-\mathrm{W}(3)-\mathrm{O}(18)$ & $156.9(9)$ & $\mathrm{O}(17)-\mathrm{W}(3)-\mathrm{O}(0 \mathrm{AA})$ & $64.0(9)$ \\
\hline $\mathrm{O}(18)-\mathrm{W}(3)-\mathrm{O}(2)$ & $63.4(8)$ & $\mathrm{O}(18)-\mathrm{W}(3)-\mathrm{O}(0 \mathrm{AA})$ & $93.9(9)$ & $\mathrm{O}(0 \mathrm{AA})-\mathrm{W}(3)-\mathrm{O}(2)$ & $42.1(8)$ \\
\hline $\mathrm{O}(8)-\mathrm{W}(4)-\mathrm{O}(9)$ & $99.7(10)$ & $\mathrm{O}(8)-\mathrm{W}(4)-\mathrm{O}(10)$ & $101.9(9)$ & $\mathrm{O}(8)-\mathrm{W}(4)-\mathrm{O}(17)$ & $100.0(8)$ \\
\hline $\mathrm{O}(8)-\mathrm{W}(4)-\mathrm{O}(19)$ & $102.2(9)$ & $\mathrm{O}(8)-\mathrm{W}(4)-\mathrm{O}(21)$ & $157.0(9)$ & $\mathrm{O}(8)-\mathrm{W}(4)-\mathrm{O}(0 \mathrm{AA})$ & $156.9(8)$ \\
\hline $\mathrm{O}(9)-\mathrm{W}(4)-\mathrm{O}(17)$ & $86.0(8)$ & $\mathrm{O}(9)-\mathrm{W}(4)-\mathrm{O}(19)$ & 158.1(9) & $\mathrm{O}(9)-\mathrm{W}(4)-\mathrm{O}(21)$ & $65.5(9)$ \\
\hline $\mathrm{O}(9)-\mathrm{W}(4)-\mathrm{O}(0 \mathrm{AA})$ & $93.4(9)$ & $\mathrm{O}(10)-\mathrm{W}(4)-\mathrm{O}(9)$ & $88.3(8)$ & $\mathrm{O}(10)-\mathrm{W}(4)-\mathrm{O}(17)$ & $158.0(9)$ \\
\hline $\mathrm{O}(10)-\mathrm{W}(4)-\mathrm{O}(19)$ & $87.6(9)$ & $\mathrm{O}(10)-\mathrm{W}(4)-\mathrm{O}(21)$ & $61.9(9)$ & $\mathrm{O}(10)-\mathrm{W}(4)-\mathrm{O}(0 \mathrm{AA})$ & $97.4(9)$ \\
\hline $\mathrm{O}(17)-\mathrm{W}(4)-\mathrm{O}(19)$ & $89.9(7)$ & $\mathrm{O}(17)-\mathrm{W}(4)-\mathrm{O}(21)$ & $96.5(9)$ & $\mathrm{O}(17)-\mathrm{W}(4)-\mathrm{O}(0 \mathrm{AA})$ & $61.9(8)$ \\
\hline $\mathrm{O}(19)-\mathrm{W}(4)-\mathrm{O}(21)$ & $93.7(9)$ & $\mathrm{O}(19)-\mathrm{W}(4)-\mathrm{O}(0 \mathrm{AA})$ & $65.9(8)$ & $\mathrm{O}(21)-\mathrm{W}(4)-\mathrm{O}(0 \mathrm{AA})$ & $45.9(8)$ \\
\hline $\mathrm{O}(9)-\mathrm{W}(5)-\mathrm{O}(20)$ & $97.0(9)$ & $\mathrm{O}(9)-\mathrm{W}(5)-\mathrm{O}(21)$ & $63.3(9)$ & $\mathrm{O}(11)-\mathrm{W}(5)-\mathrm{O}(9)$ & $87.9(9)$ \\
\hline $\mathrm{O}(11)-\mathrm{W}(5)-\mathrm{O}(15)$ & $157.1(9)$ & $\mathrm{O}(11)-\mathrm{W}(5)-\mathrm{O}(20)$ & $61.3(9)$ & $\mathrm{O}(11)-\mathrm{W}(5)-\mathrm{O}(21)$ & $95.9(9)$ \\
\hline $\mathrm{O}(12)-\mathrm{W}(5)-\mathrm{O}(9)$ & $99.9(9)$ & $\mathrm{O}(12)-\mathrm{W}(5)-\mathrm{O}(11)$ & $102.1(9)$ & $\mathrm{O}(12)-\mathrm{W}(5)-\mathrm{O}(13)$ & $101.7(9)$ \\
\hline $\mathrm{O}(12)-\mathrm{W}(5)-\mathrm{O}(15)$ & $100.8(8)$ & $\mathrm{O}(12)-\mathrm{W}(5)-\mathrm{O}(20)$ & $155.9(9)$ & $\mathrm{O}(12)-\mathrm{W}(5)-\mathrm{O}(21)$ & $155.0(8)$ \\
\hline $\mathrm{O}(13)-\mathrm{W}(5)-\mathrm{O}(9)$ & $158.4(10)$ & $\mathrm{O}(13)-\mathrm{W}(5)-\mathrm{O}(11)$ & $88.1(8)$ & $\mathrm{O}(13)-\mathrm{W}(5)-\mathrm{O}(15)$ & $87.2(9)$ \\
\hline $\mathrm{O}(13)-\mathrm{W}(5)-\mathrm{O}(20)$ & $62.7(9)$ & $\mathrm{O}(13)-\mathrm{W}(5)-\mathrm{O}(21)$ & $96.1(9)$ & $\mathrm{O}(15)-\mathrm{W}(5)-\mathrm{O}(9)$ & $88.3(8)$ \\
\hline $\mathrm{O}(15)-\mathrm{W}(5)-\mathrm{O}(20)$ & $96.9(9)$ & $\mathrm{O}(15)-\mathrm{W}(5)-\mathrm{O}(21)$ & $62.4(8)$ & $\mathrm{O}(20)-\mathrm{W}(5)-\mathrm{O}(21)$ & 49.2(9) \\
\hline $\mathrm{O}(6)-\mathrm{W}(6)-\mathrm{O}(7)$ & $100.3(9)$ & $\mathrm{O}(6)-\mathrm{W}(6)-\mathrm{O}(13)$ & $101.8(9)$ & $\mathrm{O}(6)-\mathrm{W}(6)-\mathrm{O}(14)$ & $101.5(9)$ \\
\hline $\mathrm{O}(6)-\mathrm{W}(6)-\mathrm{O}(19)$ & 101.1(9) & $\mathrm{O}(6)-\mathrm{W}(6)-\mathrm{O}(20)$ & $157.0(9)$ & $\mathrm{O}(6)-\mathrm{W}(6)-\mathrm{O}(0 \mathrm{AA})$ & $156.0(8)$ \\
\hline $\mathrm{O}(7)-\mathrm{W}(6)-\mathrm{O}(13)$ & $88.4(8)$ & $\mathrm{O}(7)-\mathrm{W}(6)-\mathrm{O}(20)$ & $63.6(9)$ & $\mathrm{O}(7)-\mathrm{W}(6)-\mathrm{O}(0 \mathrm{AA})$ & $97.9(9)$ \\
\hline $\mathrm{O}(13)-\mathrm{W}(6)-\mathrm{O}(20)$ & $63.3(9)$ & $\mathrm{O}(13)-\mathrm{W}(6)-\mathrm{O}(0 \mathrm{AA})$ & $94.2(9)$ & $\mathrm{O}(14)-\mathrm{W}(6)-\mathrm{O}(7)$ & $158.2(9)$ \\
\hline $\mathrm{O}(14)-\mathrm{W}(6)-\mathrm{O}(13)$ & $87.5(9)$ & $\mathrm{O}(14)-\mathrm{W}(6)-\mathrm{O}(20)$ & $95.6(9)$ & $\mathrm{O}(14)-\mathrm{W}(6)-\mathrm{O}(0 \mathrm{AA})$ & $61.2(8)$ \\
\hline $\mathrm{O}(19)-\mathrm{W}(6)-\mathrm{O}(7)$ & $85.9(9)$ & $\mathrm{O}(19)-\mathrm{W}(6)-\mathrm{O}(13)$ & $157.0(9)$ & $\mathrm{O}(19)-\mathrm{W}(6)-\mathrm{O}(14)$ & $89.6(8)$ \\
\hline $\mathrm{O}(19)-\mathrm{W}(6)-\mathrm{O}(20)$ & $94.3(9)$ & $\mathrm{O}(19)-\mathrm{W}(6)-\mathrm{O}(0 \mathrm{AA})$ & $64.6(8)$ & $\mathrm{O}(20)-\mathrm{W}(6)-\mathrm{O}(0 \mathrm{AA})$ & $47.0(8)$ \\
\hline $\mathrm{N}(4)-\operatorname{Ag}(1)-\mathrm{N}(4)$ & $142.3(13)$ & $\mathrm{N}(4)-\operatorname{Ag}(1)-\mathrm{N}(6)$ & $108.9(7)$ & $\mathrm{N}(1)-\operatorname{Ag}(2)-\mathrm{N}(2)$ & $120.5(5)$ \\
\hline $\mathrm{N}(2)-\operatorname{Ag}(2)-\mathrm{N}(2)$ & $118.9(9)$ & $\mathrm{N}(7)-\mathrm{Ag}(3)-\mathrm{N}(3)$ & $180.000(1$ & $\mathrm{N}(7)-\mathrm{Ag}(3)-\mathrm{N}(8)$ & $82.0(7)$ \\
\hline $\mathrm{N}(8)-\operatorname{Ag}(3)-\mathrm{N}(3)$ & $98.0(7)$ & $\mathrm{N}(8)-\mathrm{Ag}(3)-\mathrm{N}(8)$ & $164.0(14)$ & $\mathrm{N}(5)-\mathrm{Ag}(4)-\mathrm{N}(9)$ & $117.9(12)$ \\
\hline $\mathrm{N}(4)-\operatorname{Ag}(1)-\mathrm{O}(4)$ & 86.074 & $\mathrm{~N}(4)-\operatorname{Ag}(1)-\mathrm{O}(4) \# 1$ & 90.266 & $\mathrm{~N}(6)-\mathrm{Ag}(1)-\mathrm{O}(4)$ & 95.650 \\
\hline $\mathrm{N}(5)-\mathrm{Ag}(4)-\mathrm{O}(1)$ & $96.3(8)$ & $\mathrm{N}(5)-\mathrm{Ag}(4)-\mathrm{O}(8)$ & 125.504 & $\mathrm{~N}(5)-\operatorname{Ag}(4)-\mathrm{O}(22)$ & $108.8(12)$ \\
\hline $\mathrm{N}(9)-\mathrm{Ag}(4)-\mathrm{O}(1)$ & $110.1(8)$ & $\mathrm{N}(9)-\mathrm{Ag}(4)-\mathrm{O}(8)$ & 84.771 & $\mathrm{~N}(9)-\mathrm{Ag}(4)-\mathrm{O}(22)$ & $20.1(12)$ \\
\hline $\mathrm{O}(4)-\operatorname{Ag}(1)-\mathrm{O}(4)$ & 168.699 & $\mathrm{O}(1)-\mathrm{Ag}(4)-\mathrm{O}(8)$ & 123.132 & $\mathrm{O}(1)-\mathrm{Ag}(4)-\mathrm{O}(22)$ & $130.1(10)$ \\
\hline $\mathrm{O}(8)-\mathrm{Ag}(4)-\mathrm{O}(22)$ & 75.148 & & & & \\
\hline
\end{tabular}

Symmetry transformations used to generate equivalent atoms: \#1 -x,-y,-z; \#2-x+1/2,-y+1/2,-z

Table S4 Selected Bond lengths $(\AA)$ and Bond angles $\left({ }^{\circ}\right)$ of compound 3

\begin{tabular}{|l|l|l|l|l|l|}
\hline $\mathrm{P}(1)-\mathrm{O}(4)$ & $1.529(9)$ & $\mathrm{P}(1)-\mathrm{O}(4) \# 1$ & $1.529(9)$ & $\mathrm{P}(1)-\mathrm{O}(17)$ & $1.510(14)$ \\
\hline $\mathrm{P}(1)-\mathrm{O}(18)$ & $1.541(15)$ & $\mathrm{Mo}(1)-\mathrm{O}(1)$ & $1.661(9)$ & $\mathrm{Mo}(1)-\mathrm{O}(3)$ & $1.866(10)$ \\
\hline $\mathrm{Mo}(1)-\mathrm{O}(5)$ & $1.956(8)$ & $\mathrm{Mo}(1)-\mathrm{O}(7)$ & $1.888(10)$ & $\mathrm{Mo}(1)-\mathrm{O}(18)$ & $2.438(10)$ \\
\hline $\mathrm{Mo}(1)-\mathrm{O}(20)$ & $1.903(11)$ & $\mathrm{Mo}(2)-\mathrm{O}(4)$ & $2.445(10)$ & $\mathrm{Mo}(2)-\mathrm{O}(11)$ & $1.638(10)$ \\
\hline $\mathrm{Mo}(2)-\mathrm{O}(12)$ & $1.886(10)$ & $\mathrm{Mo}(2)-\mathrm{O}(14)$ & $1.904(12)$ & $\mathrm{Mo}(2)-\mathrm{O}(19)$ & $1.945(13)$ \\
\hline
\end{tabular}




\begin{tabular}{|c|c|c|c|c|c|}
\hline $\mathrm{Mo}(2)-\mathrm{O}(23)$ & $1.869(5)$ & $\mathrm{Mo}(3)-\mathrm{O}(3)$ & $1.895(10)$ & $\mathrm{Mo}(3)-\mathrm{O}(4)$ & $2.478(9)$ \\
\hline $\mathrm{Mo}(3)-\mathrm{O}(10)$ & $1.640(8)$ & $\mathrm{Mo}(3)-\mathrm{O}(13)$ & $1.906(12)$ & $\mathrm{Mo}(3)-\mathrm{O}(14)$ & $1.920(13)$ \\
\hline $\mathrm{Mo}(3)-\mathrm{O}(16)$ & $1.906(11)$ & $\mathrm{Mo}(4)-\mathrm{O}(6)$ & $1.637(10)$ & $\mathrm{Mo}(4)-\mathrm{O}(7)$ & $1.872(10)$ \\
\hline $\mathrm{Mo}(4)-\mathrm{O}(8)$ & $1.922(8)$ & $\mathrm{Mo}(4)-\mathrm{O}(9)$ & $1.892(10)$ & $\mathrm{Mo}(4)-\mathrm{O}(13)$ & $1.882(12)$ \\
\hline $\mathrm{Mo}(4)-\mathrm{O}(17)$ & $2.466(10)$ & $\mathrm{Mo}(5)-\mathrm{O}(2)$ & $1.885(4)$ & $\mathrm{Mo}(5)-\mathrm{O}(4)$ & $2.432(9)$ \\
\hline $\mathrm{Mo}(5)-\mathrm{O}(16)$ & $1.900(11)$ & $\mathrm{Mo}(5)-\mathrm{O}(19)$ & $1.912(14)$ & $\mathrm{Mo}(5)-\mathrm{O}(21)$ & $1.882(12)$ \\
\hline $\mathrm{Mo}(5)-\mathrm{O}(22)$ & $1.667(10)$ & $\mathrm{Mo}(6)-\mathrm{O}(9)$ & $1.926(11)$ & $\mathrm{Mo}(6)-\mathrm{O}(9)$ & $1.926(11)$ \\
\hline $\mathrm{Mo}(6)-\mathrm{O}(15)$ & $1.645(15)$ & $\mathrm{Mo}(6)-\mathrm{O}(17)$ & $2.443(12)$ & $\mathrm{Mo}(6)-\mathrm{O}(21)$ & $1.888(12)$ \\
\hline $\mathrm{Mo}(6)-\mathrm{O}(21)$ & $1.888(12)$ & $\mathrm{Mo}(7)-\mathrm{O}(12)$ & $1.892(11)$ & $\mathrm{Mo}(7)-\mathrm{O}(12)$ & $1.892(11)$ \\
\hline $\mathrm{Mo}(7)-\mathrm{O}(18)$ & $2.463(15)$ & $\operatorname{Mo}(7)-\mathrm{O}(20)$ & $1.905(12)$ & $\mathrm{Mo}(7)-\mathrm{O}(20)$ & $1.905(12)$ \\
\hline $\mathrm{Mo}(7)-\mathrm{O}(24)$ & $1.668(15)$ & $\operatorname{Ag}(1)-\mathrm{N}(1)$ & $2.156(10)$ & $\operatorname{Ag}(1)-\mathrm{N}(2)$ & $2.179(10)$ \\
\hline $\operatorname{Ag}(1)-\mathrm{O}(1)$ & 2.651 & $\operatorname{Ag}(1)-O(6)$ & 2.771 & $\operatorname{Ag}(1)-\mathrm{O}(11)$ & 2.687 \\
\hline $\mathrm{Ag}(1)-\mathrm{O}(22)$ & 2.727 & & & & \\
\hline $\mathrm{O}(1)-\mathrm{Mo}(1)-\mathrm{O}(3)$ & $104.6(6)$ & $\mathrm{O}(1)-\mathrm{Mo}(1)-\mathrm{O}(5)$ & $96.8(7)$ & $\mathrm{O}(1)-\mathrm{Mo}(1)-\mathrm{O}(7)$ & $104.3(6)$ \\
\hline $\mathrm{O}(1)-\mathrm{Mo}(1)-\mathrm{O}(18)$ & $162.7(6)$ & $\mathrm{O}(1)-\mathrm{Mo}(1)-\mathrm{O}(20)$ & $99.9(6)$ & $\mathrm{O}(3)-\mathrm{Mo}(1)-\mathrm{O}(5)$ & $158.6(6)$ \\
\hline $\mathrm{O}(3)-\mathrm{Mo}(1)-\mathrm{O}(7)$ & $88.7(4)$ & $\mathrm{O}(3)-\mathrm{Mo}(1)-\mathrm{O}(18)$ & $87.9(5)$ & $\mathrm{O}(3)-\mathrm{Mo}(1)-\mathrm{O}(20)$ & $87.6(5)$ \\
\hline $\mathrm{O}(5)-\mathrm{Mo}(1)-\mathrm{O}(18)$ & $71.0(5)$ & $\mathrm{O}(7)-\mathrm{Mo}(1)-\mathrm{O}(5)$ & $87.0(6)$ & $\mathrm{O}(7)-\mathrm{Mo}(1)-\mathrm{O}(18)$ & $87.7(5)$ \\
\hline $\mathrm{O}(7)-\mathrm{Mo}(1)-\mathrm{O}(20)$ & $155.6(6)$ & $\mathrm{O}(20)-\mathrm{Mo}(1)-\mathrm{O}(5)$ & $87.7(6)$ & $\mathrm{O}(20)-\mathrm{Mo}(1)-\mathrm{O}(18)$ & $68.1(5)$ \\
\hline $\mathrm{O}(11)-\mathrm{Mo}(2)-\mathrm{O}(4)$ & $163.9(6)$ & $\mathrm{O}(11)-\mathrm{Mo}(2)-\mathrm{O}(12)$ & $104.1(6)$ & $\mathrm{O}(11)-\mathrm{Mo}(2)-\mathrm{O}(14)$ & $100.6(6)$ \\
\hline $\mathrm{O}(11)-\mathrm{Mo}(2)-\mathrm{O}(19)$ & $98.5(7)$ & $\mathrm{O}(11)-\mathrm{Mo}(2)-\mathrm{O}(23)$ & $104.9(7)$ & $\mathrm{O}(12)-\mathrm{Mo}(2)-\mathrm{O}(4)$ & $88.0(4)$ \\
\hline $\mathrm{O}(12)-\mathrm{Mo}(2)-\mathrm{O}(14)$ & $87.2(5)$ & $\mathrm{O}(12)-\mathrm{Mo}(2)-\mathrm{O}(19)$ & $157.4(6)$ & $\mathrm{O}(14)-\mathrm{Mo}(2)-\mathrm{O}(4)$ & $68.9(5)$ \\
\hline $\mathrm{O}(14)-\mathrm{Mo}(2)-\mathrm{O}(19)$ & $87.3(5)$ & $\mathrm{O}(19)-\mathrm{Mo}(2)-\mathrm{O}(4)$ & $69.6(5)$ & $\mathrm{O}(23)-\mathrm{Mo}(2)-\mathrm{O}(4)$ & $85.6(6)$ \\
\hline $\mathrm{O}(23)-\mathrm{Mo}(2)-\mathrm{O}(12)$ & $89.2(6)$ & $\mathrm{O}(23)-\mathrm{Mo}(2)-\mathrm{O}(14)$ & $154.3(7)$ & $\mathrm{O}(23)-\mathrm{Mo}(2)-\mathrm{O}(19)$ & $86.4(7)$ \\
\hline $\mathrm{O}(3)-\mathrm{Mo}(3)-\mathrm{O}(4)$ & $87.0(5)$ & $\mathrm{O}(3)-\mathrm{Mo}(3)-\mathrm{O}(13)$ & $88.3(5)$ & $\mathrm{O}(3)-\mathrm{Mo}(3)-\mathrm{O}(14)$ & $87.6(5)$ \\
\hline $\mathrm{O}(3)-\mathrm{Mo}(3)-\mathrm{O}(16)$ & $154.9(5)$ & $\mathrm{O}(10)-\mathrm{Mo}(3)-\mathrm{O}(3)$ & $103.5(6)$ & $\mathrm{O}(10)-\mathrm{Mo}(3)-\mathrm{O}(4)$ & $163.6(6)$ \\
\hline $\mathrm{O}(10)-\mathrm{Mo}(3)-\mathrm{O}(13)$ & $104.6(7)$ & $\mathrm{O}(10)-\mathrm{Mo}(3)-\mathrm{O}(14)$ & $99.6(7)$ & $\mathrm{O}(10)-\mathrm{Mo}(3)-\mathrm{O}(16)$ & $101.4(7)$ \\
\hline $\mathrm{O}(13)-\mathrm{Mo}(3)-\mathrm{O}(4)$ & $88.0(5)$ & $\mathrm{O}(14)-\mathrm{Mo}(3)-\mathrm{O}(14)$ & $155.7(6)$ & $\mathrm{O}(14)-\mathrm{Mo}(3)-\mathrm{O}(4)$ & $68.0(5)$ \\
\hline $\mathrm{O}(16)-\mathrm{Mo}(3)-\mathrm{O}(4)$ & $67.9(4)$ & $\mathrm{O}(16)-\mathrm{Mo}(3)-\mathrm{O}(13)$ & $88.4(5)$ & $\mathrm{O}(16)-\mathrm{Mo}(3)-\mathrm{O}(14)$ & $85.2(5)$ \\
\hline $\mathrm{O}(6)-\mathrm{Mo}(4)-\mathrm{O}(7)$ & $105.3(6)$ & $\mathrm{O}(6)-\mathrm{Mo}(4)-\mathrm{O}(8)$ & 98.9(7) & $\mathrm{O}(6)-\mathrm{Mo}(4)-\mathrm{O}(9)$ & $99.5(6)$ \\
\hline $\mathrm{O}(6)-\mathrm{Mo}(4)-\mathrm{O}(13)$ & $104.3(6)$ & $\mathrm{O}(6)-\mathrm{Mo}(4)-\mathrm{O}(17)$ & $162.1(5)$ & $\mathrm{O}(7)-\mathrm{Mo}(4)-\mathrm{O}(8)$ & $87.0(6)$ \\
\hline $\mathrm{O}(7)-\mathrm{Mo}(4)-\mathrm{O}(9)$ & $155.0(6)$ & $\mathrm{O}(7)-\mathrm{Mo}(4)-\mathrm{O}(13)$ & $89.1(5)$ & $\mathrm{O}(7)-\mathrm{Mo}(4)-\mathrm{O}(17)$ & $87.4(5)$ \\
\hline $\mathrm{O}(8)-\mathrm{Mo}(4)-\mathrm{O}(17)$ & $68.7(6)$ & $\mathrm{O}(9)-\mathrm{Mo}(4)-\mathrm{O}(8)$ & $86.7(6)$ & $\mathrm{O}(9)-\mathrm{Mo}(4)-\mathrm{O}(17)$ & $67.8(5)$ \\
\hline $\mathrm{O}(13)-\mathrm{Mo}(4)-\mathrm{O}(8)$ & $156.7(7)$ & $\mathrm{O}(13)-\mathrm{Mo}(4)-\mathrm{O}(9)$ & $87.2(6)$ & $\mathrm{O}(13)-\mathrm{Mo}(4)-\mathrm{O}(17)$ & $88.1(5)$ \\
\hline $\mathrm{O}(2)-\mathrm{Mo}(5)-\mathrm{O}(4)$ & $85.6(5)$ & $\mathrm{O}(2)-\mathrm{Mo}(5)-\mathrm{O}(16)$ & $154.6(6)$ & $\mathrm{O}(2)-\mathrm{Mo}(5)-\mathrm{O}(19)$ & $86.4(6)$ \\
\hline $\mathrm{O}(16)-\mathrm{Mo}(5)-\mathrm{O}(4)$ & $69.1(4)$ & $\mathrm{O}(16)-\mathrm{Mo}(5)-\mathrm{O}(19)$ & $87.5(5)$ & $\mathrm{O}(19)-\mathrm{Mo}(5)-\mathrm{O}(4)$ & $70.3(5)$ \\
\hline $\mathrm{O}(21)-\mathrm{Mo}(5)-\mathrm{O}(2)$ & $87.3(6)$ & $\mathrm{O}(21)-\mathrm{Mo}(5)-\mathrm{O}(4)$ & $88.0(5)$ & $\mathrm{O}(21)-\mathrm{Mo}(5)-\mathrm{O}(16)$ & $89.2(5)$ \\
\hline $\mathrm{O}(21)-\mathrm{Mo}(5)-\mathrm{O}(19)$ & $157.8(6)$ & $\mathrm{O}(22)-\mathrm{Mo}(5)-\mathrm{O}(2)$ & $105.6(6)$ & $\mathrm{O}(22)-\mathrm{Mo}(5)-\mathrm{O}(4)$ & $164.0(5)$ \\
\hline $\mathrm{O}(22)-\mathrm{Mo}(5)-\mathrm{O}(16)$ & $99.7(6)$ & $\mathrm{O}(22)-\mathrm{Mo}(5)-\mathrm{O}(19)$ & $98.6(6)$ & $\mathrm{O}(22)-\mathrm{Mo}(5)-\mathrm{O}(21)$ & $103.6(6)$ \\
\hline $\mathrm{O}(9)-\mathrm{Mo}(6)-\mathrm{O}(9)$ & $85.9(7)$ & $\mathrm{O}(9)-\mathrm{Mo}(6)-\mathrm{O}(17)$ & $67.8(4)$ & $\mathrm{O}(9)-\mathrm{Mo}(6)-\mathrm{O}(17)$ & $67.8(4)$ \\
\hline $\mathrm{O}(15)-\mathrm{Mo}(6)-\mathrm{O}(9)$ & $101.2(6)$ & $\mathrm{O}(15)-\mathrm{Mo}(6)-\mathrm{O}(9)$ & $101.2(6)$ & $\mathrm{O}(15)-\mathrm{Mo}(6)-\mathrm{O}(17)$ & $164.4(8)$ \\
\hline $\mathrm{O}(15)-\mathrm{Mo}(6)-\mathrm{O}(21)$ & $103.4(7)$ & $\mathrm{O}(15)-\mathrm{Mo}(6)-\mathrm{O}(21)$ & $103.4(7)$ & $\mathrm{O}(21)-\mathrm{Mo}(6)-\mathrm{O}(9)$ & $155.3(6)$ \\
\hline $\mathrm{O}(21)-\mathrm{Mo}(6)-\mathrm{O}(9)$ & $88.1(5)$ & $\mathrm{O}(21)-\mathrm{Mo}(6)-\mathrm{O}(9)$ & $88.1(5)$ & $\mathrm{O}(21)-\mathrm{Mo}(6)-\mathrm{O}(9)$ & $155.3(6)$ \\
\hline $\mathrm{O}(21)-\mathrm{Mo}(6)-\mathrm{O}(17)$ & $87.7(5)$ & $\mathrm{O}(21)-\mathrm{Mo}(6)-\mathrm{O}(17)$ & $87.7(5)$ & $\mathrm{O}(21)-\mathrm{Mo}(6)-\mathrm{O}(21)$ & $87.4(8)$ \\
\hline $\mathrm{O}(12)-\mathrm{Mo}(7)-\mathrm{O}(12)$ & $89.6(7)$ & $\mathrm{O}(12)-\mathrm{Mo}(7)-\mathrm{O}(18)$ & $87.3(5)$ & $\mathrm{O}(12)-\mathrm{Mo}(7)-\mathrm{O}(18)$ & $87.3(5)$ \\
\hline $\mathrm{O}(12)-\mathrm{Mo}(7)-\mathrm{O}(20)$ & $154.7(5)$ & $\mathrm{O}(12)-\mathrm{Mo}(7)-\mathrm{O}(20)$ & $154.7(5)$ & $\mathrm{O}(12)-\mathrm{Mo}(7)-\mathrm{O}(20)$ & $86.7(5)$ \\
\hline
\end{tabular}




\begin{tabular}{|l|l|l|l|l|l|}
\hline $\mathrm{O}(12)-\mathrm{Mo}(7)-\mathrm{O}(20)$ & $86.6(5)$ & $\mathrm{O}(20)-\mathrm{Mo}(7)-\mathrm{O}(18)$ & $67.5(4)$ & $\mathrm{O}(20)-\mathrm{Mo}(7)-\mathrm{O}(18)$ & $67.5(4)$ \\
\hline $\mathrm{O}(20)-\mathrm{Mo}(7)-\mathrm{O}(20)$ & $86.2(6)$ & $\mathrm{O}(24)-\mathrm{Mo}(7)-\mathrm{O}(12)$ & $104.8(6)$ & $\mathrm{O}(24)-\mathrm{Mo}(7)-\mathrm{O}(12)$ & $104.8(6)$ \\
\hline $\mathrm{O}(24)-\mathrm{Mo}(7)-\mathrm{O}(18)$ & $162.7(8)$ & $\mathrm{O}(24)-\mathrm{Mo}(7)-\mathrm{O}(20)$ & $100.4(7)$ & $\mathrm{O}(24)-\mathrm{Mo}(7)-\mathrm{O}(20)$ & $100.4(7)$ \\
\hline $\mathrm{N}(1)-\mathrm{Ag}(1)-\mathrm{N}(2)$ & $169.5(4)$ & $\mathrm{N}(1)-\mathrm{Ag}(1)-\mathrm{O}(1)$ & 101.047 & $\mathrm{~N}(1)-\mathrm{Ag}(1)-\mathrm{O}(6)$ & 82.385 \\
\hline $\mathrm{N}(1)-\mathrm{Ag}(1)-\mathrm{O}(11)$ & 86.943 & $\mathrm{~N}(1)-\mathrm{Ag}(1)-\mathrm{O}(22)$ & 87.184 & $\mathrm{~N}(2)-\mathrm{Ag}(1)-\mathrm{O}(1)$ & 86.337 \\
\hline $\mathrm{N}(2)-\mathrm{Ag}(1)-\mathrm{O}(6)$ & 88.352 & $\mathrm{~N}(2)-\mathrm{Ag}(1)-\mathrm{O}(11)$ & 85.81 & $\mathrm{~N}(2)-\mathrm{Ag}(1)-\mathrm{O}(22)$ & 102 \\
\hline $\mathrm{O}(1)-\mathrm{Ag}(1)-\mathrm{O}(6)$ & 162.566 & $\mathrm{O}(1)-\operatorname{Ag}(1)-\mathrm{O}(11)$ & 87.792 & $\mathrm{O}(1)-\mathrm{Ag}(1)-\mathrm{O}(22)$ & 76.274 \\
\hline $\mathrm{O}(6)-\mathrm{Ag}(1)-\mathrm{O}(11)$ & 75.257 & $\mathrm{O}(6)-\operatorname{Ag}(1)-\mathrm{O}(22)$ & 121.109 & $\mathrm{O}(11)-\mathrm{Ag}(1)-\mathrm{O}(22)$ & 161.618 \\
\hline
\end{tabular}

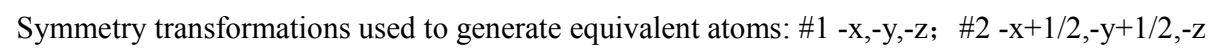

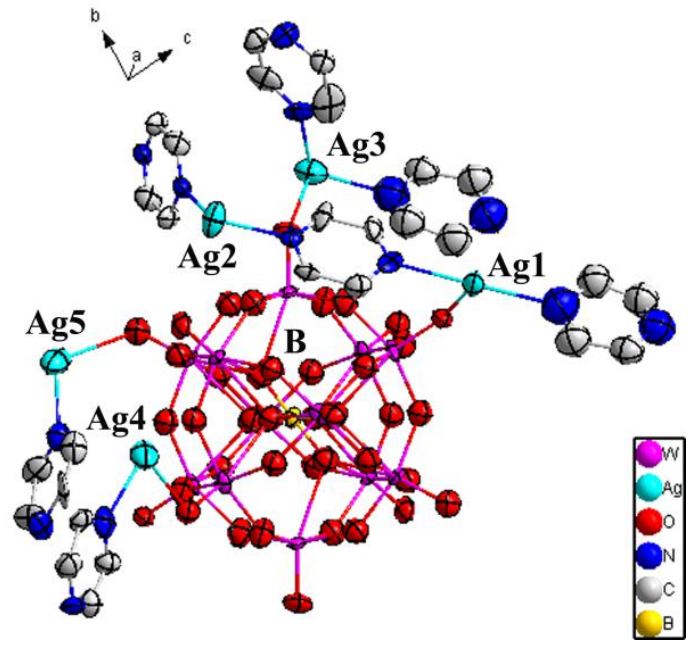

Figure S1. The ellipsoid structures of the compounds 1.

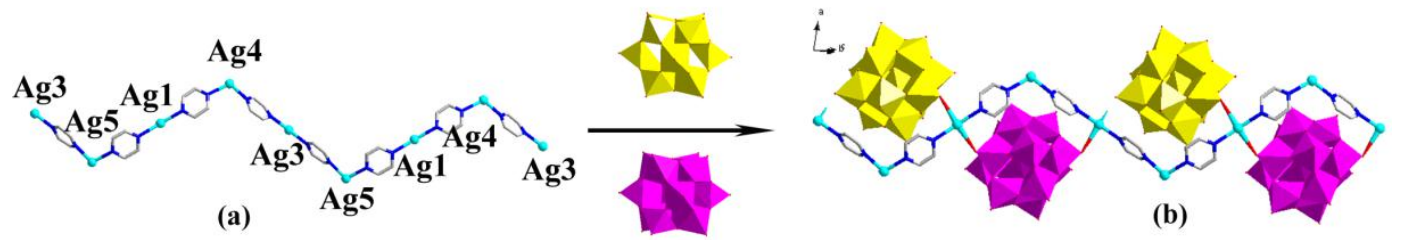

Figure S2. Structure of compound 1. (a) The Ag-pz curve-like chains;(b) View of the 1D chain;

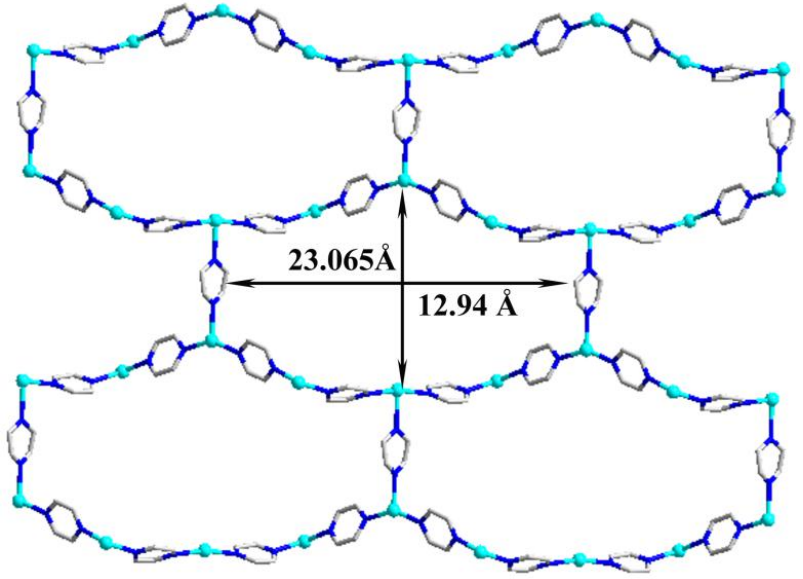

Figure S3. The 2D Ag-pz layerof compound 1 


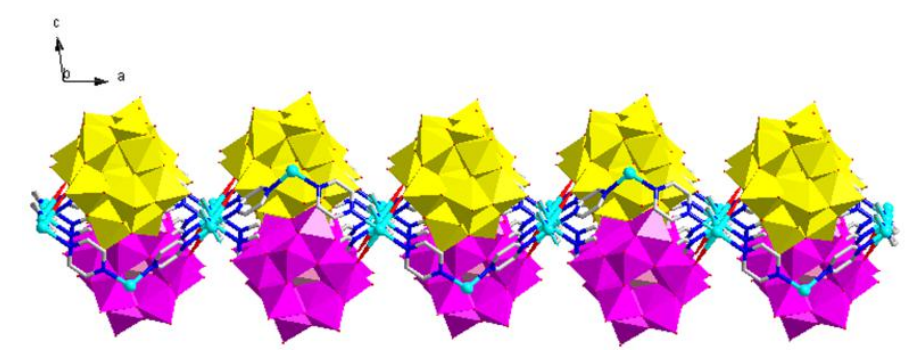

Figure S4. View of the location of $\left\{\mathrm{BW}_{12}\right\}$ anions in 2D layer of compound 1.

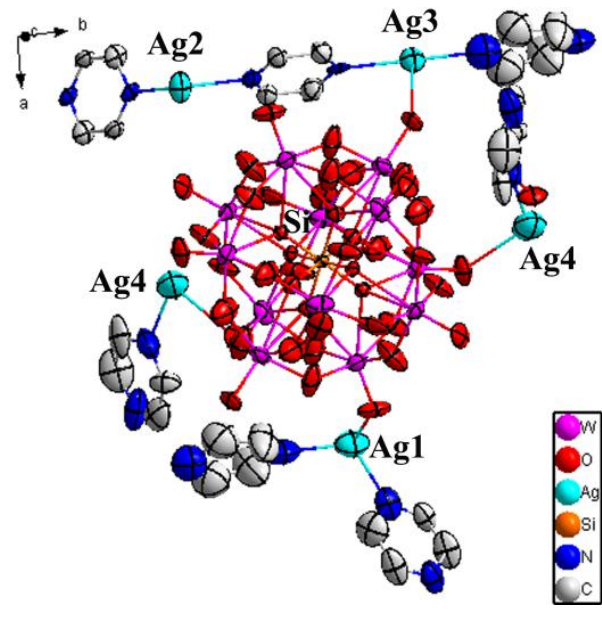

Figure S5. The ellipsoid structures of the compounds 2.

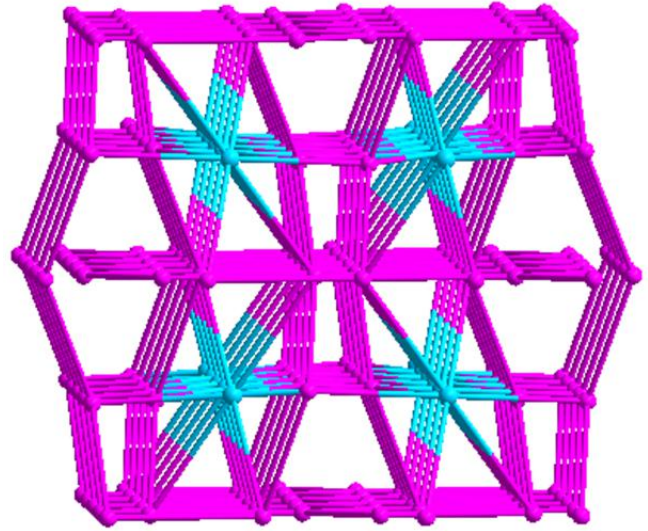

Figure S6. Topology structure of compound 2.

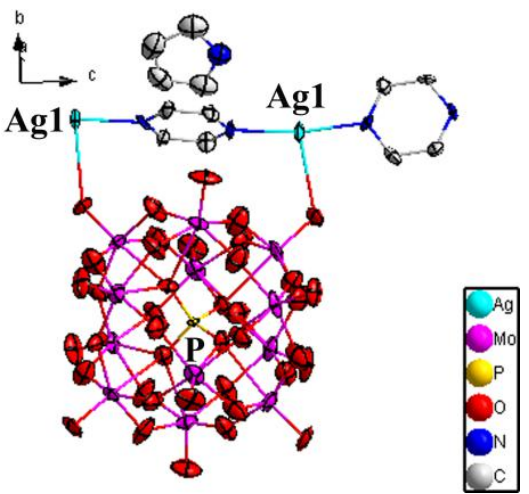

Figure S7. The ellipsoid structures of the compounds 3. 


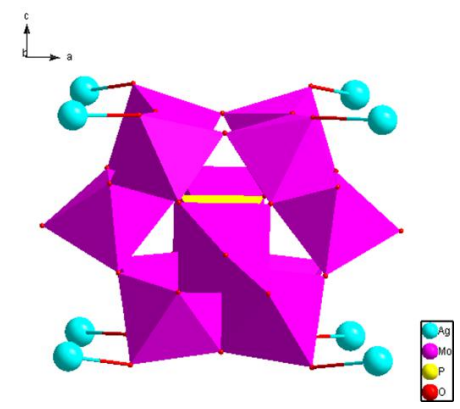

Figure S8. The eight-dentate coordination mode of $\left\{\mathrm{PMo}_{12}\right\}$ anion.

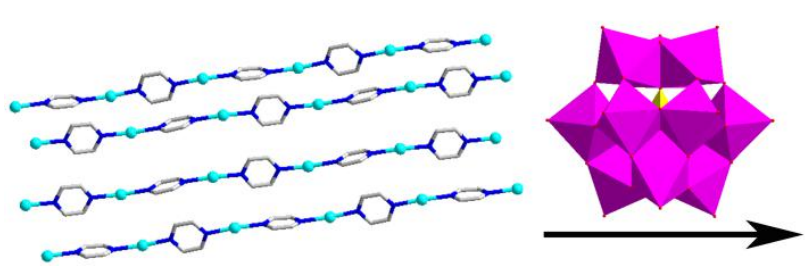

(a)

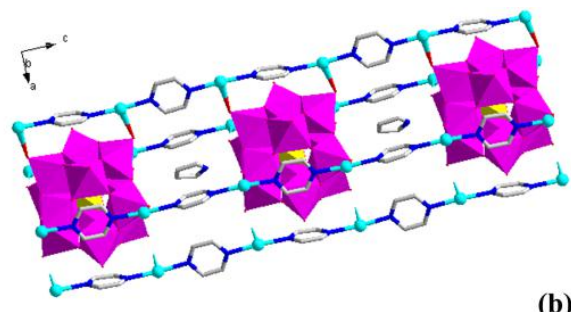

(b)

Figure S9. (a) View of the Ag-pz chainof compound 3; (b) view of the 1D chain.

(a)

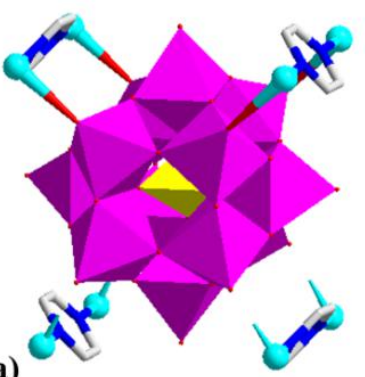

(b)

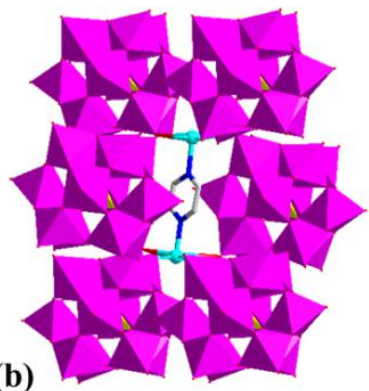

Figure S10. (a) view of the coordination modes of the anions $\left\{\mathrm{PMo}_{12}\right\}$ of compound 3 ; (b) view of the coordination modes of the $\left\{\mathrm{Ag}_{2}(\mathrm{pz})\right\}$.
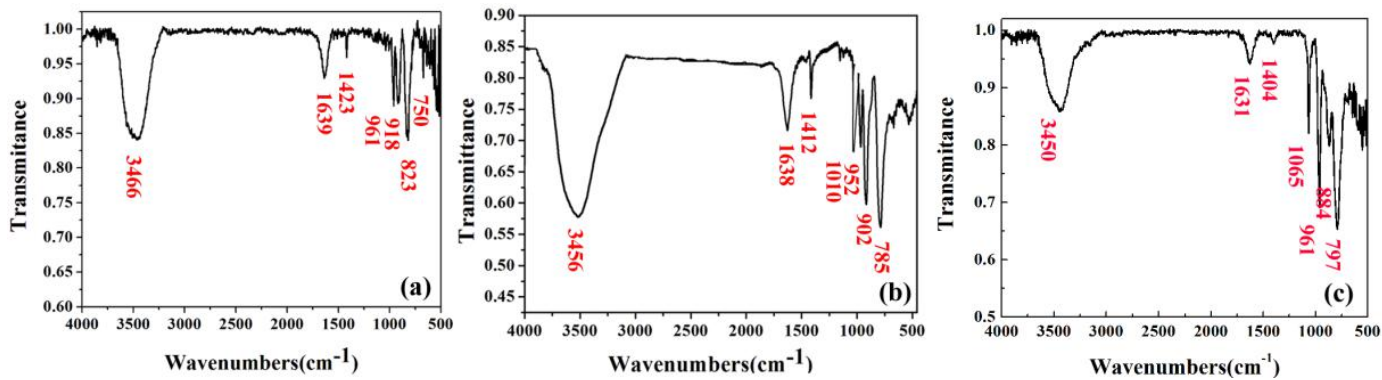

Figure S11. The FT-IR spectrum in the range $4000-500 \mathrm{~cm}^{-1}$ for compounds 1-3.
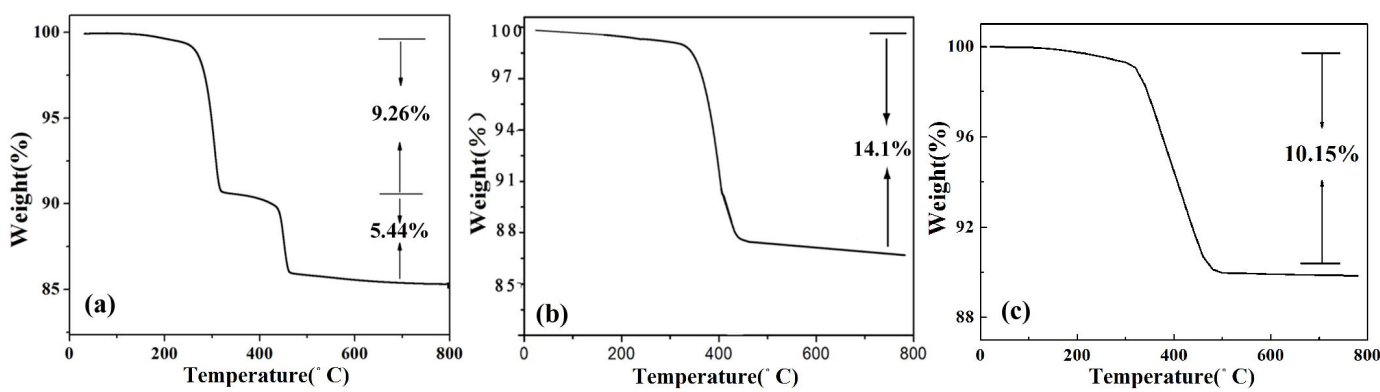

Figure S12. The TG curve for compounds 1-3. 

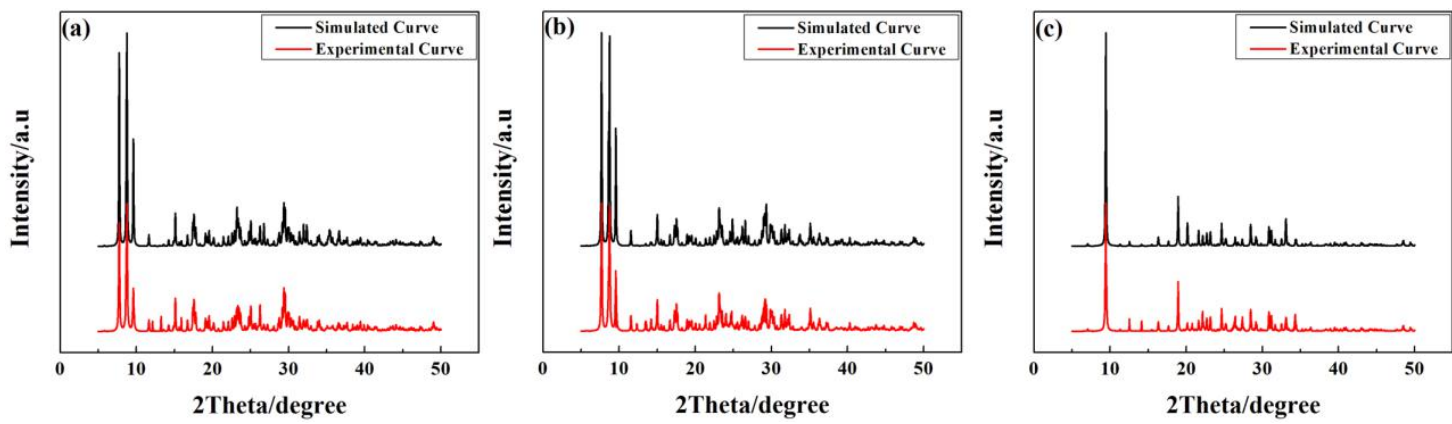

Figure S13. The PXRD contrast curves of compounds 1-3.

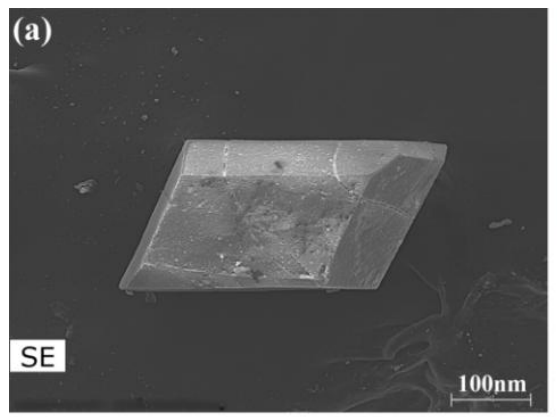

(b)
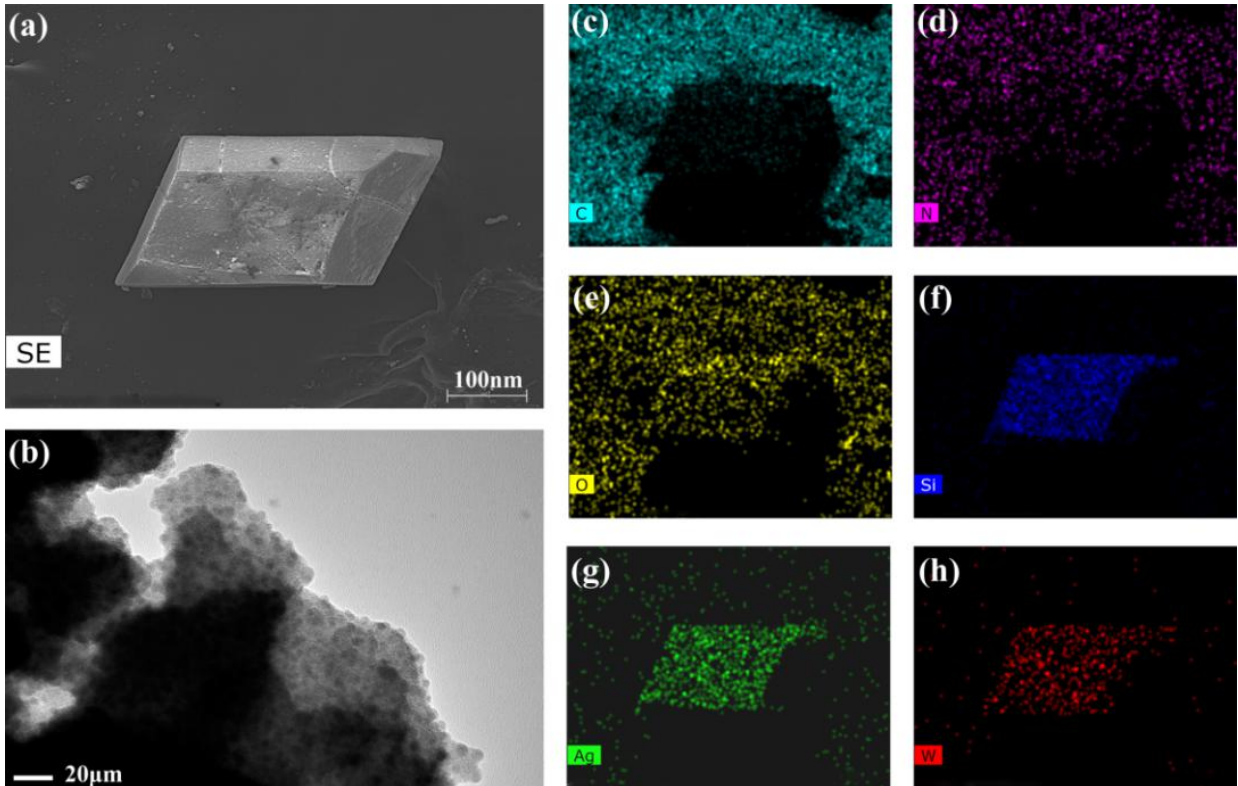

Figure S14. The morphology images of compound 2. (a) SEM images; (b) TEM images; (c-h) EDS elemental mappings of O, C, Si, N, Ag, and W for compound 2.
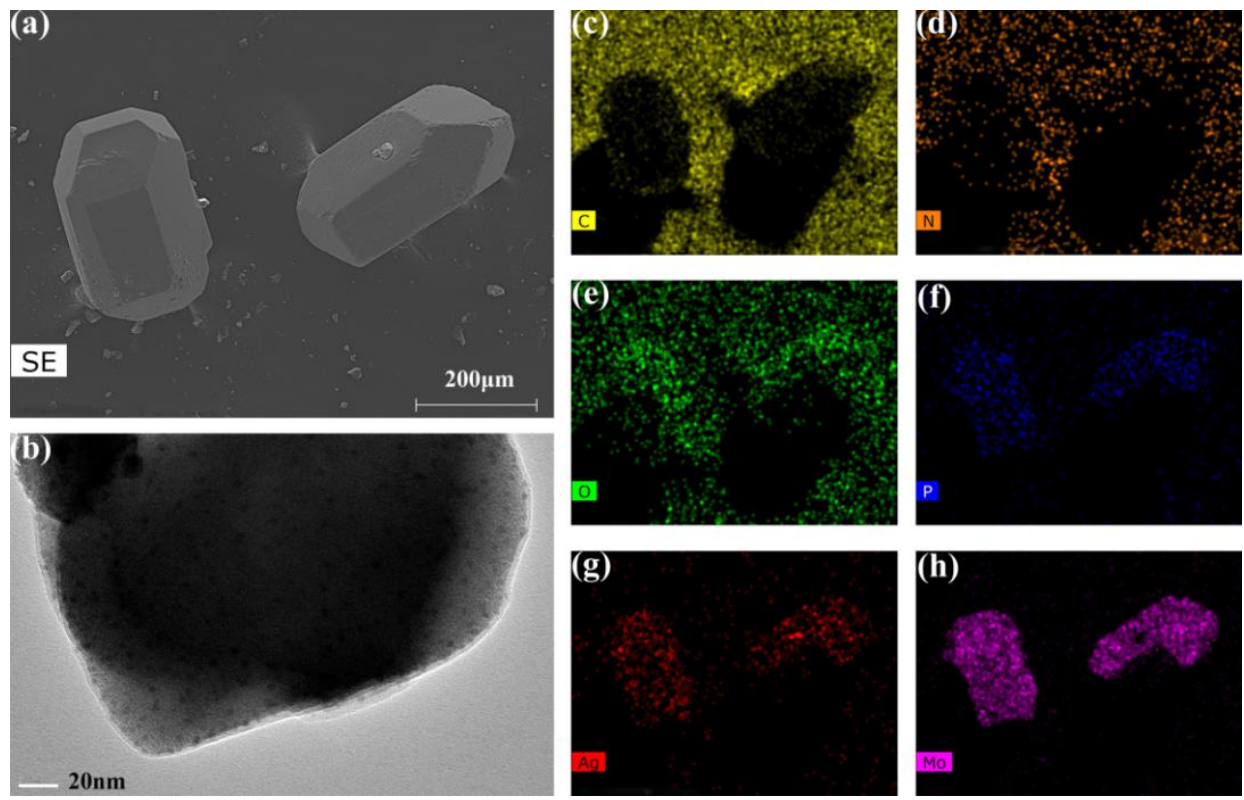

Figure S15. The morphology images of compound 3. (a) SEM images; (b) TEM images; (c-h) EDS elemental mappings of O, C, P, N, Ag, and Mo for compound 3. 

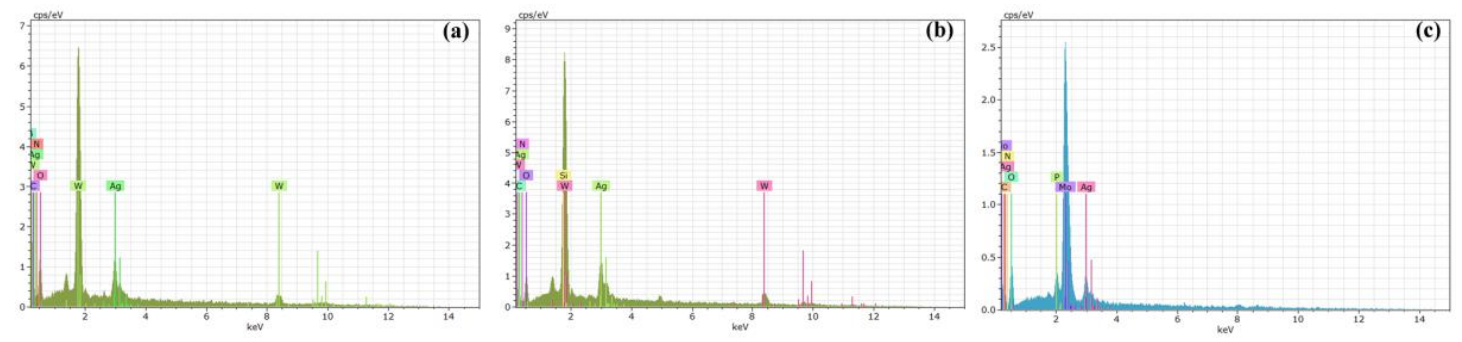

Figure S16. (a) The EDS microanalysis of the compound 1; (b) The EDS microanalysis of the compound 2; (c) The EDS microanalysis of the compound 3.
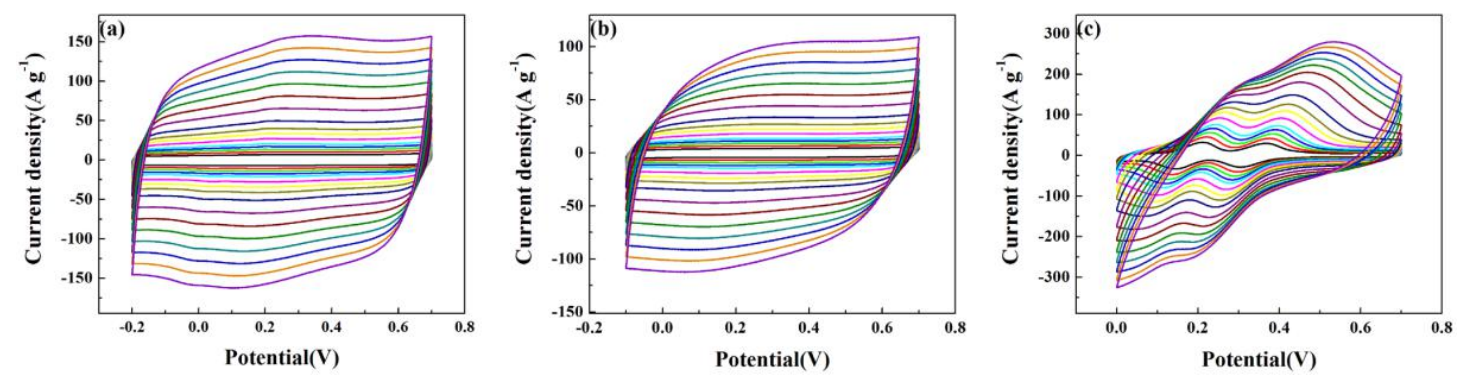

Figure S17. CV curves of the $\mathrm{BW}_{12}$-based, $\mathrm{SiW}_{12}$-based and $\mathrm{PMo}_{12}$-based electrodes in $1 \mathrm{M} \mathrm{H}_{2} \mathrm{SO}_{4}$ solution recorded at scan rates of $20,30,40,50,60,80,100,120,150$, $200,250,300,350,400,450$ and $500 \mathrm{mV} \mathrm{s}^{-1}$.
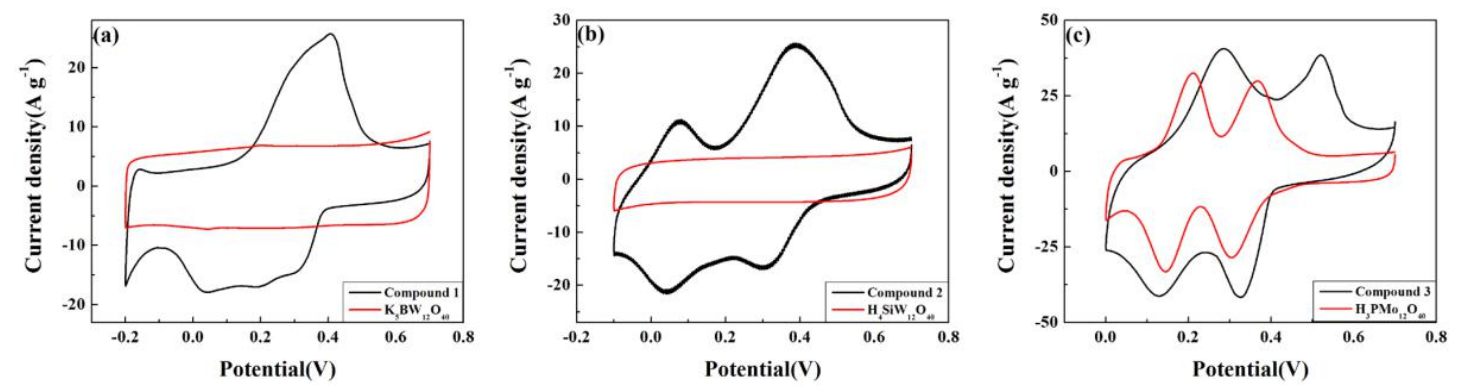

Figure S18. (a) CV curves for $\mathrm{BW}_{12}$-based and 1-based electrodes at $20 \mathrm{mV} \mathrm{s}^{-1}$. (b) $\mathrm{CV}$ curves for $\mathrm{SiW}_{12}$-based and 2-based electrodes at $20 \mathrm{mV} \mathrm{s}^{-1}$. (c) $\mathrm{CV}$ curves for

$\mathrm{PMo}_{12}$-based and 3-based electrodes at $20 \mathrm{mV} \mathrm{s}^{-1}$.
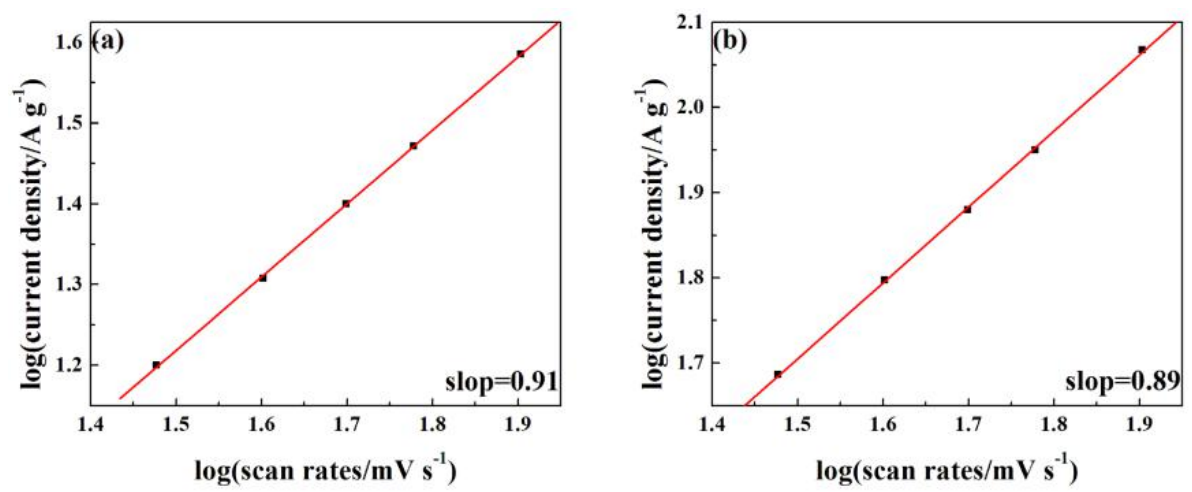

Figure S19. The plot of $\log$ of current density vs. the log of scan rate in the scan rate range of 30-80 $\mathrm{mV} \mathrm{s}^{-1}$ for 2-based and 3-based electrodes. 

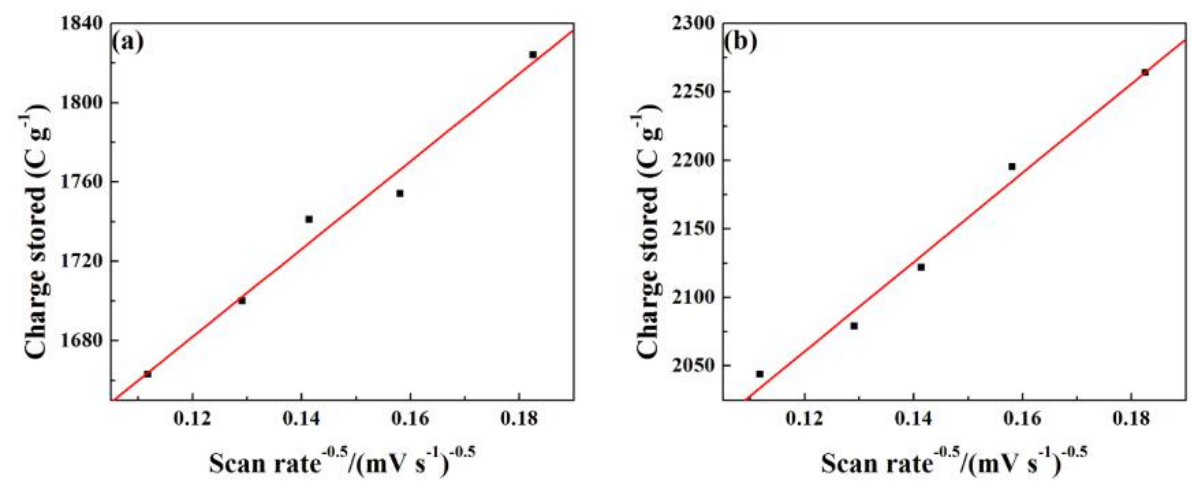

Figure S20. The plot of the total charge stored (q) vs. the reciprocal of the square root of the scan rate for 2-based and 3-based electrodes.
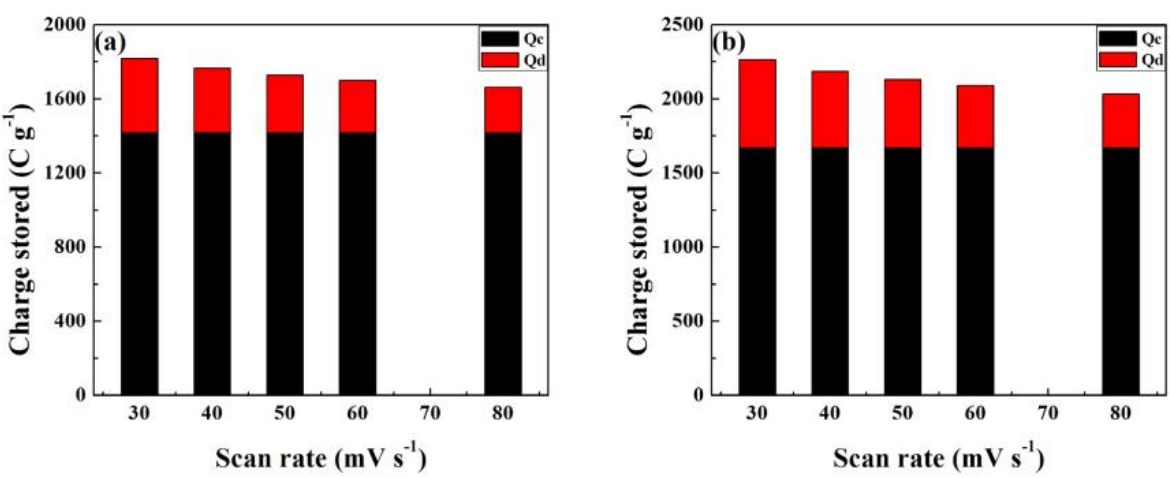

Figure S21. Normalized contribution ratio of the capacitive (Qs) and diffusion-controlled (Qd) charge storage capacities at lower scan rates.
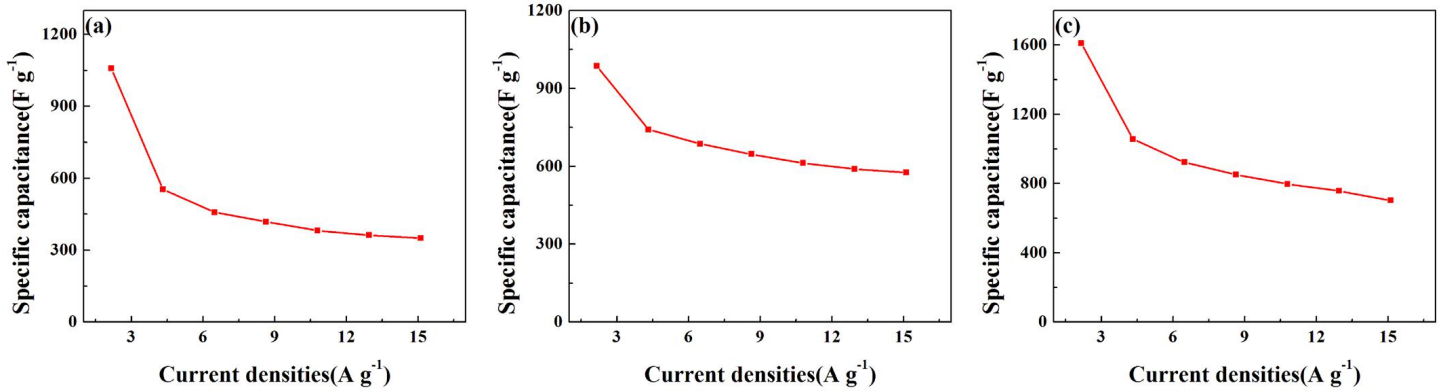

Figure S22. The good rate capabilities of these electrodes at the different current density.
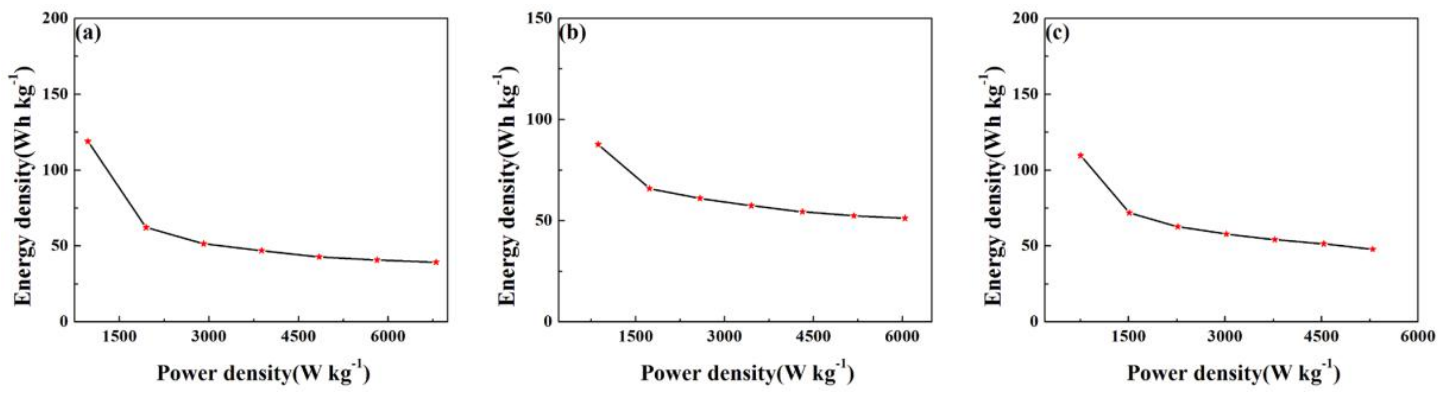

Figure S23. Ragone plots for 1-based, 2-based and 3-based electrodes. 

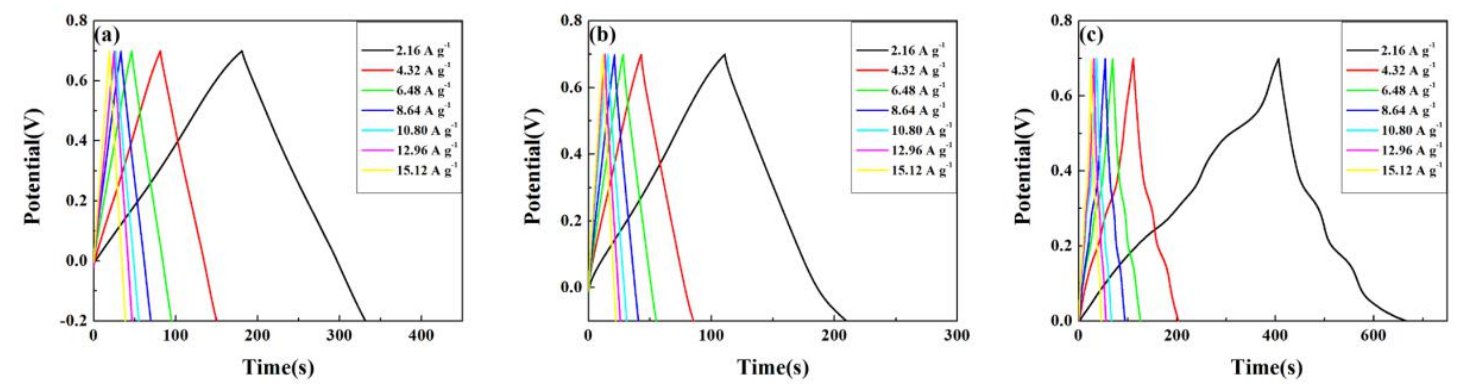

Figure S24. GCD curves for the $\mathrm{BW}_{12}$-based, $\mathrm{SiW}_{12}$-based and $\mathrm{PMo}_{12}$-based electrodes at the current density of $2.16 \mathrm{~A} \mathrm{~g}^{-1}, 4.32 \mathrm{~A} \mathrm{~g}^{-1}, 6.48 \mathrm{~A} \mathrm{~g}^{-1}, 8.64 \mathrm{~A} \mathrm{~g}^{-1}, 10.8 \mathrm{~A}$ $\mathrm{g}^{-1}, 12.96 \mathrm{~A} \mathrm{~g}^{-1}, 15.12 \mathrm{~A} \mathrm{~g}^{-1}$.
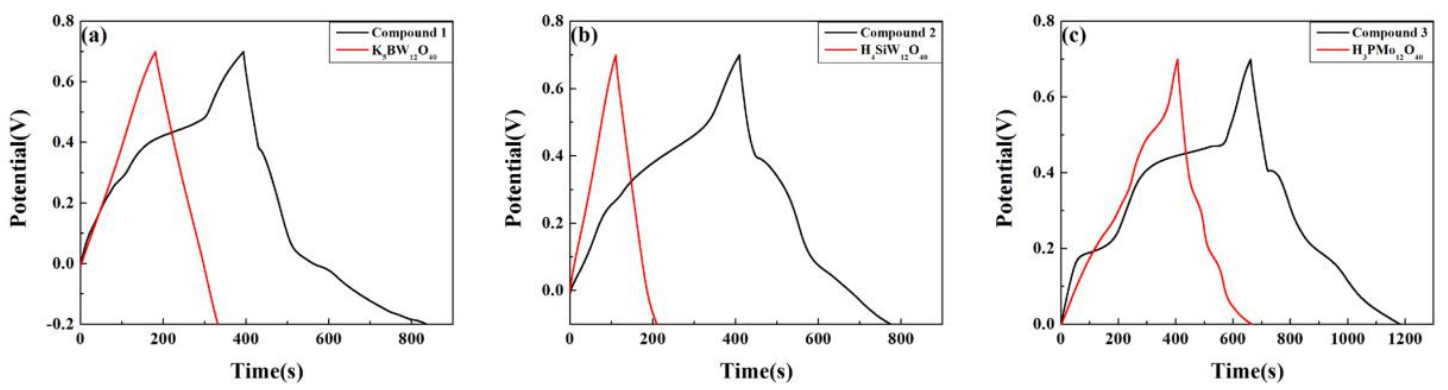

Figure S25. (a)GCD curves for 1-based and BW 12 -based electrodes, recorded at a current density of $2.16 \mathrm{~A} \mathrm{~g}^{-1}$. (b) GCD curves for 2-based and $\mathrm{SiW}_{12}$-based electrodes, recorded at a current density of 2.16 $\mathrm{A} \mathrm{g}^{-1}$.(c) GCD curves for 3-based and

$\mathrm{PMo}_{12}$-based electrodes, recorded at a current density of $2.16 \mathrm{~A} \mathrm{~g}^{-1}$.

(a)

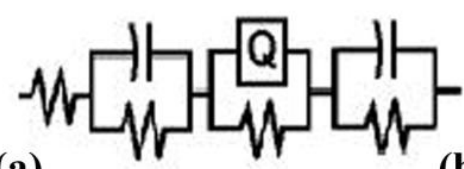

(b)

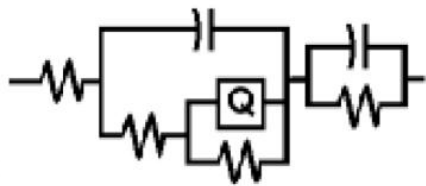

(c)

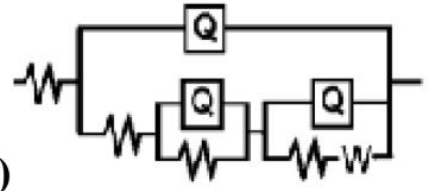

Figure S26. The equivalent electric circuit for 1-based, 2-based and 3-based
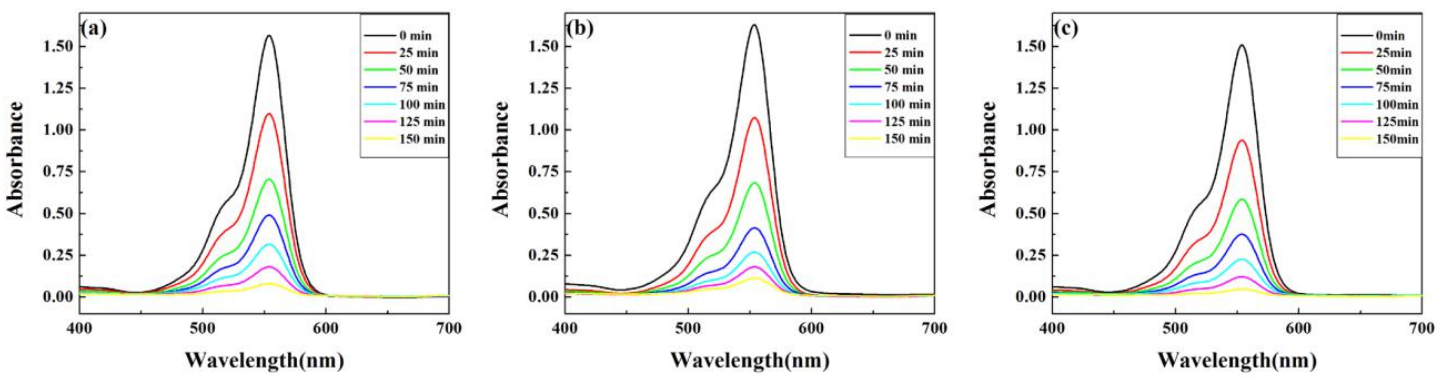

Figure S27. Absorption spectras of $\mathrm{RhB}$ solution during the decomposition reaction under UV irradiation in the presence of compounds 1-3. 

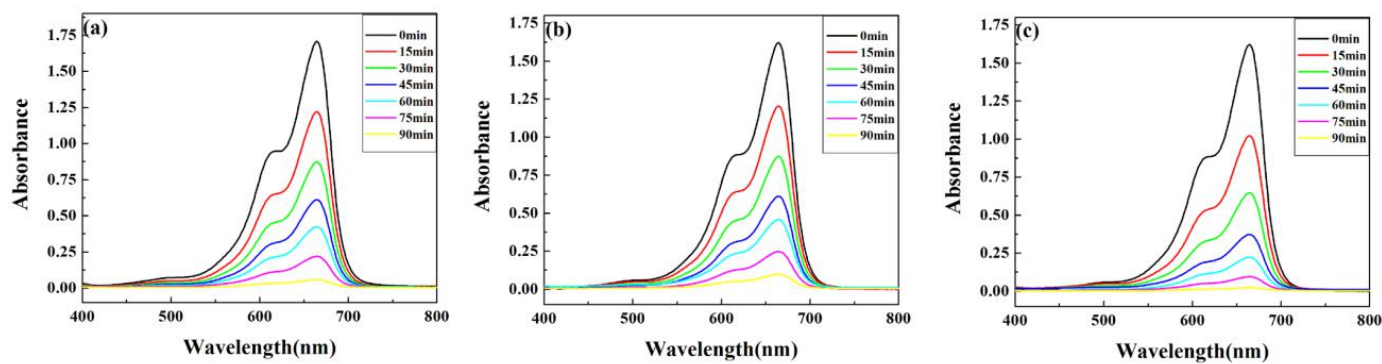

Figure S28. Absorption spectras of MB solution during the decomposition reaction under UV irradiation in the presence of compounds 1-3.
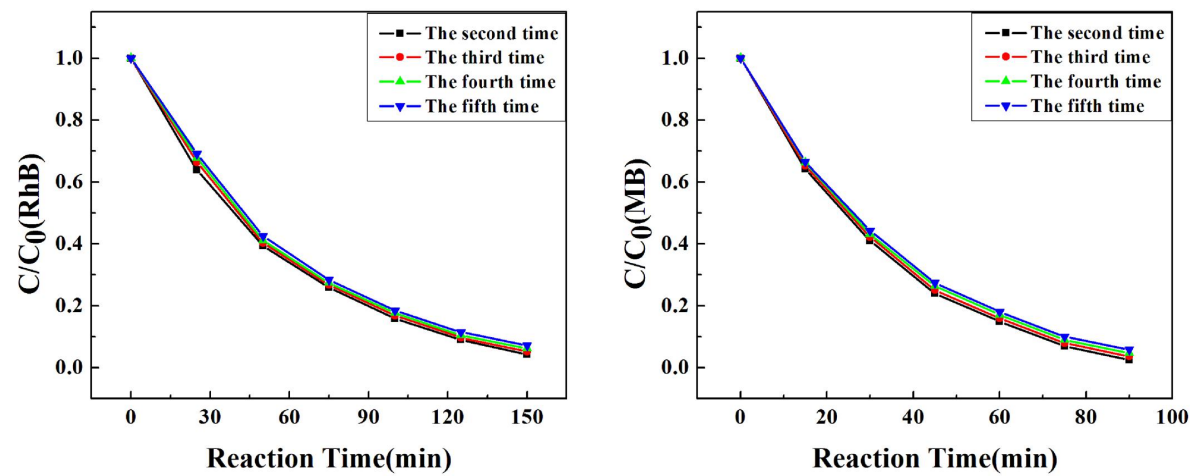

Figure S29. Plot of reaction time vs. the concentration of dyestuff with compounds 1-3. (a) $\mathrm{RhB}$ (b) $\mathrm{MB}$

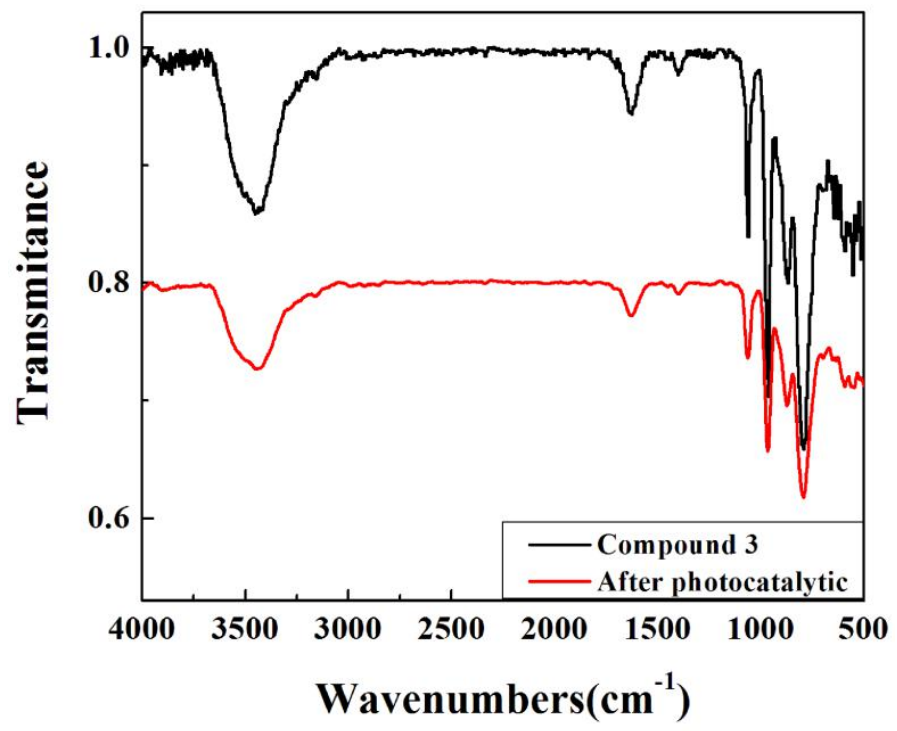

Figure S30. The IR spectra of the compounds $\mathbf{3}$ before and after recycling reactions.

Table S5 Other POMs-based electrode materials.

\begin{tabular}{|l|l|l|l|l|}
\hline Type & Electrolyte & $\begin{array}{l}\text { Scan rate/ } \\
\text { Current } \\
\text { density }\end{array}$ & $\begin{array}{l}\text { Specific } \\
\text { capacitance }\end{array}$ & Ref \\
\hline $\mathrm{MoS} / \mathrm{rGO}$ & $0.5 \mathrm{M} \mathrm{Na}_{2} \mathrm{SO}_{4}+\mathrm{H}_{2} \mathrm{SO}_{4}$ & $10 \mathrm{mV} \mathrm{s}^{-1}$ & $870 \mathrm{~F} \mathrm{~g}^{-1}$ & {$[1]$} \\
\hline$\left[\mathrm{Ru}(\mathrm{bpy})_{3}\right] 3.33 \mathrm{PMo}_{18} \mathrm{O}_{62} \mathrm{mH}_{2} \mathrm{O}$ & $\begin{array}{l}\mathrm{pH}=7(0.25 \mathrm{M} \text { total salt } \\
\text { containing } 0.05 \mathrm{M} \\
\mathrm{KH}_{2} \mathrm{PO}_{4}, 0.05 \mathrm{M}\end{array}$ & $0.2 \mathrm{~A} \mathrm{~g}^{-1}$ & $125 \mathrm{~F} \mathrm{~g}^{-1}$ & {$[2]$} \\
\hline
\end{tabular}




\begin{tabular}{|c|c|c|c|c|}
\hline & $\begin{array}{l}\mathrm{K}_{2} \mathrm{HPO}_{4}, 0.1 \mathrm{M} \mathrm{NaCl} \\
0.025 \mathrm{M} \mathrm{MgCl}_{2} \text { and } \\
\left.0.025 \mathrm{M} \mathrm{CaCl}_{2}\right)\end{array}$ & & & \\
\hline$\left[\mathrm{Ru}(\mathrm{bpy})_{3}\right]_{3} \mathrm{PMo}_{18} \mathrm{O}_{62} \cdot \mathrm{nH}_{2} \mathrm{O}$ & - & $0.2 \mathrm{~A} \mathrm{~g}^{-1}$ & $68 \mathrm{~F} \mathrm{~g}^{-1}$ & {$[2]$} \\
\hline $\mathrm{AC} @ \mathrm{PMo}_{12}$ & $1 \mathrm{M}[\mathrm{Bmim}] \mathrm{H}_{2} \mathrm{SO}_{4}$ & $1 \mathrm{mV} \mathrm{s}^{-1}$ & $223 \mathrm{~F} \mathrm{~g}^{-1}$ & {$[3]$} \\
\hline HPW/RGO & $5 \mathrm{M} \mathrm{H}_{2} \mathrm{SO}_{4}$ & $5 \mathrm{mV} \mathrm{s}^{-1}$ & $337.5 \mathrm{~F} \mathrm{~g}^{-1}$ & {$[4]$} \\
\hline$\left[\mathrm{PW}_{11} \mathrm{MO}_{39}\right]^{5-} @ \mathrm{Ru}-\mathrm{rGO}(\mathrm{M}: \mathrm{Co})$ & $0.5 \mathrm{M}$ HOAC. & $0.2 \mathrm{~A} \mathrm{~g}^{-1}$ & $624 \mathrm{~F} \mathrm{~g}^{-1}$ & [5] \\
\hline$\left[\mathrm{PW}_{11} \mathrm{MO}_{39}\right]^{5-} @ \mathrm{Ru}-\mathrm{rGO}(\mathrm{M}: \mathrm{Ni})$ & $0.5 \mathrm{M}$ HOAC. & $0.2 \mathrm{~A} \mathrm{~g}^{-1}$ & $568 \mathrm{~F} \mathrm{~g}^{-1}$ & {$[5]$} \\
\hline$\left[\mathrm{PW}_{11} \mathrm{MO}_{39}\right]^{5-} @ \mathrm{Ru}-\mathrm{rGO}(\mathrm{M}: \mathrm{Cu})$ & $0.5 \mathrm{M}$ HOAC. & $0.2 \mathrm{~A} \mathrm{~g}^{-1}$ & $705 \mathrm{~F} \mathrm{~g}^{-1}$ & {$[5]$} \\
\hline $\mathrm{AC} / \mathrm{P}_{2} \mathrm{Mo}_{18}$ & $1 \mathrm{M} \mathrm{H}_{2} \mathrm{SO}_{4}$ & $6 \mathrm{~A} \mathrm{~g}^{-1}$ & $275 \mathrm{~F} \mathrm{~g}^{-1}$ & {$[6]$} \\
\hline $\mathrm{AC} / \mathrm{PMo}_{12}$ & $1 \mathrm{M} \mathrm{H}_{2} \mathrm{SO}_{4}$ & $2 \mathrm{~A} \mathrm{~g}^{-1}$ & $\begin{array}{l}160 \mathrm{~F}^{-1} \text { and } \\
183 \mathrm{~F} \mathrm{~g}^{-1} \text { for the } \\
\text { positive and } \\
\text { negative hybrid } \\
\text { electrodes, }\end{array}$ & {$[7]$} \\
\hline POM-Ppy/nanopillar & $0.05 \mathrm{M} \mathrm{H}_{2} \mathrm{SO}_{4}$ & $1 \mathrm{~mA} \mathrm{~cm}^{-2}$ & $77.0 \mathrm{mF} \mathrm{cm}^{-2}$ & {$[8]$} \\
\hline $\mathrm{AC}-\mathrm{PW} \mathrm{W}_{12}$ & $1 \mathrm{M} \mathrm{H}_{2} \mathrm{SO}_{4}$ & $10 \mathrm{mV} \mathrm{s}^{-1}$ & $254 \mathrm{~F} \mathrm{~g}^{-1}$ & [9] \\
\hline HT-RGO-PMo 12 & $1 \mathrm{M} \mathrm{H}_{2} \mathrm{SO}_{4}$ & $10 \mathrm{mV} \mathrm{s}^{-1}$ & $276 \mathrm{~F} \mathrm{~g}^{-1}$ & [10] \\
\hline $\mathrm{PPy}-\mathrm{PMo}_{12} / \mathrm{rGO} \mathrm{TNHs}$ & $0.5 \mathrm{M} \mathrm{H}_{2} \mathrm{SO}_{4}$ & $0.5 \mathrm{~A} \mathrm{~g}^{-1}$ & $360 \mathrm{~F} \mathrm{~g}^{-1}$ & [11] \\
\hline PMoW-PDDA-RGO & $1 \mathrm{M} \mathrm{H}_{2} \mathrm{SO}_{4}$ & $1 \mathrm{Ag} \mathrm{g}^{-1}$ & $279.1 \mathrm{~F} \mathrm{~g}^{-1}$ & [12] \\
\hline Pinecone $\mathrm{AC} / \mathrm{PMo}_{12} \mathrm{O}_{40}$ & $1 \mathrm{M} \mathrm{H}_{2} \mathrm{SO}_{4}$ & $10 \mathrm{mV} \mathrm{s}^{-1}$ & $\begin{array}{l}361 \mathrm{~F} \mathrm{~g}^{-1}(1.19 \mathrm{~F} \\
\left.\mathrm{cm}^{-2}\right)\end{array}$ & [13] \\
\hline $\mathrm{Na}_{6} \mathrm{~V}_{10} \mathrm{O}_{28}$ & $\begin{array}{l}1 \mathrm{~m} \mathrm{LiClO}_{4} \text { in propylene } \\
\text { carbonate }\end{array}$ & $0.1 \mathrm{~A} \mathrm{~g}^{-1}$ & $354 \mathrm{~F} \mathrm{~g}^{-1}$ & [14] \\
\hline $\mathrm{AC} / \mathrm{PMo}_{12}$ & $1 \mathrm{M} \mathrm{H}_{2} \mathrm{SO}_{4}$ & $10 \mathrm{mV} \mathrm{s}^{-1}$ & $246 \mathrm{~F} \mathrm{~g}^{-1}$ & [15] \\
\hline $\mathrm{PIL} / \mathrm{POM} / \mathrm{RGO}$ & $0.5 \mathrm{M} \mathrm{H}_{2} \mathrm{SO}_{4}$ & $10 \mathrm{mV} \mathrm{s}^{-1}$ & $456 \mathrm{~F} \mathrm{~g} \mathrm{~g}^{-1}$ & [16] \\
\hline$\left[\mathrm{Ag}_{5}(\mathrm{brtmb})_{4}\right]\left[{ }^{\mathrm{V}} \mathrm{W}_{10} \mathrm{~V}_{2} \mathrm{O}_{40}\right]$ & $1 \mathrm{M} \mathrm{H}_{2} \mathrm{SO}_{4}$ & $110 \mathrm{~A} \mathrm{~g}^{-1}$ & $206 \mathrm{~F} \mathrm{~g}^{-1}$ & [17] \\
\hline $\begin{array}{l}\left(\mathrm{H}_{2} \text { bpe }\right)(\mathrm{Hbpe})_{2}\left\{\left[\mathrm{Cu}(\mathrm{pzta})\left(\mathrm{H}_{2} \mathrm{O}\right)\right]\left[\mathrm{P}_{2}\right.\right. \\
\left.\left.\mathrm{W}_{18} \mathrm{O}_{62}\right]\right\} \cdot 5 \mathrm{H}_{2} \mathrm{O}\end{array}$ & $1 \mathrm{M} \mathrm{H}_{2} \mathrm{SO}_{4}$ & $5 \mathrm{~A} \mathrm{~g}^{-1}$ & $168 \mathrm{~F} \mathrm{~g}^{-1}$ & [18] \\
\hline$\left(\mathrm{PMo}_{12} / \mathrm{PANI} / \mathrm{TiN} \mathrm{NWA}\right)$ & $1 \mathrm{M} \mathrm{H}_{2} \mathrm{SO}_{4}$ & $1 \mathrm{~A} \mathrm{~g}^{-1}$ & $469 \mathrm{~F} \mathrm{~g}^{-1}$ & [19] \\
\hline Compound 1 & $1 \mathrm{M} \mathrm{H}_{2} \mathrm{SO}_{4}$ & $2.16 \mathrm{Ag}^{-1}$ & $1058 \mathrm{~F} \mathrm{~g}^{-1}$ & \\
\hline Compound 2 & $1 \mathrm{M} \mathrm{H}_{2} \mathrm{SO}_{4}$ & $2.16 \mathrm{~A} \mathrm{~g}^{-1}$ & $986 \mathrm{~F} \mathrm{~g}^{-1}$ & \\
\hline Compound 3 & $1 \mathrm{M} \mathrm{H}_{2} \mathrm{SO}_{4}$ & $2.16 \mathrm{Ag}^{-1}$ & $1611 \mathrm{~F} \mathrm{~g}^{-1}$ & \\
\hline
\end{tabular}

Table S6 Other MOF-based electrode materials.

\begin{tabular}{|l|l|l|l|l|}
\hline Type & Electrolyte & $\begin{array}{l}\text { Scan rate/ Current } \\
\text { density }\end{array}$ & Specific & Ref \\
\hline Capacitance $\left(\mathrm{F} \mathrm{g} \mathrm{g}^{-1}\right)$ & \\
\hline POAP/ZIF-67 & $0.1 \mathrm{M} \mathrm{HClO}_{4}$ & $0.005 \mathrm{~mA}$ & 724 & {$[20]$} \\
\hline
\end{tabular}




\begin{tabular}{|c|c|c|c|c|}
\hline Ni- DMOF- ADC & $2 \mathrm{M} \mathrm{KOH}$ & $1 \mathrm{~A} / \mathrm{g}$ & 552 & [22] \\
\hline Co-MOF & $1 \mathrm{M} \mathrm{LiOH}$ & $0.6 \mathrm{~A} / \mathrm{g}$ & 207 & [23] \\
\hline Co8-MOF-5 & $\begin{array}{l}0.1 \mathrm{M} \\
\text { TBATF }_{6}\end{array}$ & $10 \mathrm{~mA} / \mathrm{g}$ & 0.3 & [24] \\
\hline Co-BPDC & $0.5 \mathrm{M} \mathrm{LiOH}$ & $10 \mathrm{mV} / \mathrm{s}$ & 179 & [25] \\
\hline $\mathrm{Ni}_{3}(\mathrm{HITP})_{2}$ & $\mathrm{TEABF}_{4}$ & $50 \mathrm{mV} / \mathrm{s}$ & 111 & [26] \\
\hline PANI-ZIF67-CC & $3 \mathrm{M} \mathrm{KCl}$ & $10 \mathrm{mV} / \mathrm{s}$ & 371 & [27] \\
\hline $\begin{array}{l}\text { PANI-CNT@ZIF-6 } \\
\text { 7-CC }\end{array}$ & $3 \mathrm{M} \mathrm{KCl}$ & $10 \mathrm{mV} / \mathrm{s}$ & 0.58 & [28] \\
\hline ZIF-PPy-2 & $1 \mathrm{M} \mathrm{Na}_{2} \mathrm{SO}_{4}$ & $0.5 \mathrm{~A} / \mathrm{g}$ & 554 & [29] \\
\hline Ni-MOF & $2 \mathrm{M} \mathrm{KOH}$ & $1 \mathrm{~A} / \mathrm{g}$ & 726 & {$[30]$} \\
\hline $\mathrm{Cu}-\mathrm{MOF} / \mathrm{rGO}$ & $0.5 \mathrm{M} \mathrm{Na}_{2} \mathrm{SO}_{4}$ & $1 \mathrm{~A} / \mathrm{g}$ & 385 & {$[31]$} \\
\hline Zn-MOF & $1 \mathrm{M} \mathrm{Li}_{2} \mathrm{SO}_{4}$ & $2.5 \mathrm{~mA} / \mathrm{g}$ & 23 & [32] \\
\hline Zn-MOF/PANi & $1 \mathrm{M} \mathrm{H}_{2} \mathrm{SO}_{4}$ & $1 \mathrm{~A} / \mathrm{g}$ & 477 & [33] \\
\hline $\mathrm{Cu}-\mathrm{MOF}$ & $1 \mathrm{M} \mathrm{Na}_{2} \mathrm{SO}_{4}$ & $1.6 \mathrm{~A} / \mathrm{g}$ & 85 & {$[34]$} \\
\hline Compound 1 & $1 \mathrm{M} \mathrm{H}_{2} \mathrm{SO}_{4}$ & $2.16 \mathrm{~A} \mathrm{~g}^{-1}$ & $1058 \mathrm{~F} \mathrm{~g}^{-1}$ & \\
\hline Compound 2 & $1 \mathrm{M} \mathrm{H}_{2} \mathrm{SO}_{4}$ & $2.16 \mathrm{~A} \mathrm{~g}^{-1}$ & $986 \mathrm{~F} \mathrm{~g}^{-1}$ & \\
\hline Compound 3 & $1 \mathrm{M} \mathrm{H}_{2} \mathrm{SO}_{4}$ & $2.16 \mathrm{~A} \mathrm{~g}^{-1}$ & $1611 \mathrm{~F} \mathrm{~g}^{-1}$ & \\
\hline
\end{tabular}

Table S7 Some classical $\left\{\mathrm{BW}_{12}\right\},\left\{\mathrm{SiW}_{12}\right\}$ and $\left\{\mathrm{PMo}_{12}\right\}$ structure photocatalytic degradation rate summary

\begin{tabular}{|c|c|c|c|c|c|c|c|}
\hline \multirow[b]{2}{*}{ compound } & \multicolumn{3}{|c|}{$\mathrm{RhB}$} & \multicolumn{3}{|c|}{ MB } & \multirow[t]{2}{*}{ literature } \\
\hline & $\mathrm{T} / \mathrm{min}$ & $\begin{array}{l}\text { rates } \\
/ \%\end{array}$ & lamp & $\mathrm{T} / \mathrm{min}$ & $\begin{array}{l}\text { rates } \\
/ \%\end{array}$ & lamp & \\
\hline Compound 1 & 150 & 94.89 & UV & 90 & 96.48 & UV & \\
\hline Compound 2 & 150 & 93.06 & UV & 90 & 94.28 & UV & \\
\hline Compound 3 & 150 & 96.88 & UV & 90 & 98.58 & UV & \\
\hline $\begin{array}{c}{\left[\mathrm{Cu}_{2} \mathrm{~L}_{2}\left(\mathrm{PMo}^{\mathrm{VI}}{ }_{11} \mathrm{Mo}^{\mathrm{V}} \mathrm{O}_{40}\right)\left(\mathrm{H}_{2} \mathrm{O}\right)_{2}\right] \cdot 2 \mathrm{H}_{2}} \\
\mathrm{O}\end{array}$ & & & & 210 & 35.9 & UV & [35] \\
\hline$\left[\mathrm{Cu}_{2} \mathrm{~L}_{2}\left(\mathrm{SiW}_{12} \mathrm{O}_{40}\right)\left(\mathrm{H}_{2} \mathrm{O}\right)_{6}\right] \cdot \mathrm{H}_{2} \mathrm{O}$ & & & & 210 & 90.6 & UV & [35] \\
\hline $\left.\mathrm{Ag}_{4}\left(\mathrm{~L}^{1}\right)_{2}\left(\mathrm{HSiW}^{\mathrm{VI}}{ }_{11} \mathrm{~V}^{\mathrm{V}} \mathrm{O}_{40}\right)\right] \cdot 25 \mathrm{H}_{2} \mathrm{O}$ & & & & 120 & 93.0 & UV & [36] \\
\hline $\begin{array}{c}{\left[\mathrm{Ag}_{3}(4-\mathrm{ptz})_{2} \mathrm{Na}(\text { phen })\left(\mathrm{HPMo}_{12} \mathrm{O}_{40}\right)\right] \cdot} \\
2 \mathrm{H}_{2} \mathrm{O}\end{array}$ & & & & 180 & 73.6 & UV & [37] \\
\hline$\left[\mathrm{Ag}_{4}(3-\text { ptz })_{2}(\text { phen })_{2}\left(\mathrm{HPMo}_{12} \mathrm{O}_{40}\right)\right]$ & & & & 180 & 82.3 & UV & [37] \\
\hline$\left[\mathrm{Ag}_{3}(3\right.$-bptzb $\left.)(\text { phen })_{2}\left(\mathrm{PMo}_{12} \mathrm{O}_{40}\right)\right]$ & & & & 180 & 56.6 & UV & [37] \\
\hline$\left[\mathrm{Ag}_{10} \mathrm{~L}_{8}{ }_{8}\left(\mathrm{HPMo}_{12} \mathrm{O}_{40}\right)\right] \cdot \mathrm{H}_{2} \mathrm{O}$ & 120 & 60 & UV & 120 & 51 & UV & [38] \\
\hline $\begin{array}{c}{\left[\mathrm{Co}^{\mathrm{II}}(\mathrm{HBBTZ})(\mathrm{BBTZ})_{2.5}\right]} \\
{\left[\mathrm{PMo}_{12} \mathrm{O}_{40}\right]}\end{array}$ & & & & 90 & 93.6 & UV & [39] \\
\hline$\left[\mathrm{Cu}^{\mathrm{I}}(\mathrm{BBTZ})\right]_{5}\left[\mathrm{BW}_{12} \mathrm{O}_{40}\right] \cdot \mathrm{H}_{2} \mathrm{O}$ & & & & 90 & 90.9 & UV & [39] \\
\hline$\left[\mathrm{Ag}_{2}\left(\mathrm{~L}^{1}\right)_{4}\right]\left[\mathrm{HPMo}_{12} \mathrm{O}_{40}\right] \cdot 3 \mathrm{H}_{2} \mathrm{O}$ & 100 & 25.04 & UV & 100 & 80.79 & UV & [40] \\
\hline
\end{tabular}




\begin{tabular}{|c|c|c|c|c|c|c|c|}
\hline$\left[\mathrm{Cu}_{6}^{\mathrm{I}} \mathrm{Cl}_{4}\left(\mathrm{~L}^{2}\right)_{6}\right]\left[\mathrm{H}_{3} \mathrm{PMo}_{12} \mathrm{O}_{40}\right] \cdot 2 \mathrm{NO}_{3}$ & 100 & 20.08 & UV & 100 & 83.21 & UV & [40] \\
\hline $\mathrm{Zn}(\mathrm{Htib})(\mathrm{tib}) \mathrm{PMo}_{12} \mathrm{O}_{40}$ & 120 & 76.5 & UV & & & & [41] \\
\hline $\begin{array}{c}{\left[\mathrm{H}_{2} \mathrm{bimb}_{1.5}\left[\mathrm{Co}(\mathrm{bimb})_{1.5}\left(\mathrm{H}_{2} \mathrm{O}\right)\right.\right.} \\
\left.\left(\mathrm{BW}_{12} \mathrm{O}_{40}\right)\right] \cdot 4 \mathrm{H}_{2} \mathrm{O}\end{array}$ & & & & 60 & 92.6 & UV & [42] \\
\hline $\begin{array}{c}{\left[\mathrm{H}_{2} \mathrm{bimb}\right]_{1.5}\left[\mathrm{Ni}(\mathrm{bimb})_{1.5}\left(\mathrm{H}_{2} \mathrm{O}\right)\left(\mathrm{BW}_{12}\right.\right.} \\
\left.\left.\mathrm{O}_{40}\right)\right] \cdot 4 \mathrm{H}_{2} \mathrm{O}\end{array}$ & & & & 60 & 93.4 & UV & [42] \\
\hline $\begin{array}{l}\mathrm{K}\left[\mathrm{H}_{2} \mathrm{bimb}\right]\left[\mathrm{Cu}(\mathrm{bimb})\left(\mathrm{BW}_{12} \mathrm{O}_{40}\right)\right] \cdot 2 \mathrm{H} \\
{ }_{2} \mathrm{O}\end{array}$ & & & & 60 & 93.4 & UV & [42] \\
\hline $\begin{array}{c}{\left[\mathrm{Cu}_{12}^{\mathrm{I}}(\operatorname{trz})_{8}\left(\mathrm{H}_{2} \mathrm{O}\right)_{2}\right]\left[\alpha-\mathrm{SiW}_{12} \mathrm{O}_{40}\right] \cdot 2 \mathrm{H}_{2}} \\
\mathrm{O}\end{array}$ & 240 & 91 & UV & & & & [43] \\
\hline$\left[\mathrm{Co}\left(\mathrm{L}^{1}\right)_{2}\left(\mathrm{H}_{2} \mathrm{O}\right)_{2}\right]_{2}\left[\mathrm{SiW}_{12} \mathrm{O}_{40}\right] \cdot 6 \mathrm{H}_{2} \mathrm{O}$ & 90 & 94.18 & UV & & & & [44] \\
\hline $\begin{array}{c}{\left[\mathrm{Cu}_{2}\left(\mathrm{C}_{9} \mathrm{H}_{22}\right.\right.} \\
\left.\mathrm{N}_{2}\left(\mathrm{~L}^{2}\right)_{2}\right]\left[\mathrm{SiW}_{12} \mathrm{O}_{40}\right] \cdot 2 \mathrm{H}_{2} \mathrm{O}\end{array}$ & 90 & 92.04 & UV & & & & [44] \\
\hline $\begin{array}{c}\left(\mathrm{H}_{2} \mathrm{bibp}\right)_{2}\left[\mathrm{bibp}\{\mathrm{Cu}(\mathrm{bipy})(\mathrm{bibp})\}_{2}\{\mathrm{Cu}\right. \\
\left.(\text { bipy }) \mathrm{Cl}\}_{2}\left\{\mathrm{BW}_{12} \mathrm{O}_{40}\right\}_{2}\right] \cdot 3 \mathrm{H}_{2} \mathrm{O}\end{array}$ & 180 & 94.18 & UV & 175 & 95.32 & UV & [45] \\
\hline $\begin{array}{c}{\left[\left\{\mathrm{Cu}(\text { bipy })_{2}\right\}\{\mathrm{Cu}(\text { bipy })(\mathrm{Hbtb})\}\left\{\mathrm{BW}_{1}\right.\right.} \\
\left.\left.{ }_{2} \mathrm{O}_{40}\right\}\right]\end{array}$ & 180 & 90.64 & UV & 175 & 95.67 & UV & [45] \\
\hline
\end{tabular}

1. Zhang, S.; Liu, R.; Li, S.; Dolbecq, A.; Mialane, P.; Suo, L.; Bi, L.; Zhang, B.; Liu, T.; Wu, C.; Yan, L.; Su, Z.; Zhang, G.; Keita, B. Simple and efficient polyoxomolybdate-mediated synthesis of novel graphene and metal nanohybrids for versatile applications. J. Colloid Interf. Sci. 2018, 514, 507-516.

2. Chinnathambi, S.; Ammam, M. A molecular hybrid polyoxometalate-organometallic moieties and its relevance to supercapacitors in physiological electrolytes. J. Power Sources 2015, 284, 524-535.

3. Hu, C.; Zhao, E.; Nitta, N.; Magasinski, A.; Berdichevsky, G.; Yushin, G. Aqueous solutions ofacidic ionic liquids for enhanced stability of polyoxometalate-carbon supercapacitor electrodes. J. Power Sources, 2016, 326, $569-574$. 
4. Li, R.; He, C.; Cheng, L.; Lin, G.; Wang, G.; Shi, D.; Li, R.-K.; Yang, Y. Polyoxometalate-enabled photoreduction of graphene oxide to bioinspired nacre-like composite films for supercapacitor electrodes. Composites Part B: Engineering, 2017, 121, 75-82.

5. Ensafi, A.-A.; Heydari-Soureshjani, E.; Rezaei, B. $\left[\mathrm{PW}_{11} \mathrm{MO}_{39}\right]^{5-}$ decorated on Ru-reduced graphene oxide nanosheets, characterizations and application as a high performance storage energy andoxygen reduction reaction. Chem. Eng. J. 2017, 330, 1109-1118.

6. Mu, A.; Li, J.; Chen, W.; Sang, X.; Su, Z.; Wang, E. The composite material based on Dawson-type polyoxometalate and activated carbon as the supercapacitor electrode. Inorg. Chem. Commun. 2015, 55, 149-152.

7. Ruiz, V.; Suárez-Guevara, J.; Gomez-Romero, P. Hybrid electrodes based on polyoxometalate-carbon materials for electrochemical supercapacitors. Electrochem. Commun. 2012, 24, 35-38.

8. Yang, M. H.; Hong, S. B.; Yoon, J. H.; Kim, D. S.; Jeong, S. W.; Yoo, D. E.; Lee, T. J.; Lee, K. G.; Lee, S. J.; Choi, B. G. Fabrication of Flexible, Redoxable, and Conductive Nanopillar Arrays with Enhanced Electrochemical Performance. ACS Appl. Mater. Interfaces, 2016, 8, 22220-22226. 
9. Suárez-Guevara, J.; Ruiz, V.; Gomez-Romero, P. Hybrid energy storage: high voltage aqueous supercapacitors based on activated carbon-phosphotungstate hybrid materials. J. Mater. Chem. A, 2014, 2, 1014-1021.

10. Suarez-Guevara, J.; Ruiz, $\quad$ V.; Gomez-Romero, $\quad$ P. $\quad$ Stable graphene-polyoxometalate nanomaterials for application in hybrid supercapacitors. Phys. Chem. Chem. Phys. 2014, 16, 20411-20414.

11. Chen, Y.; Han, M.; Tang, Y.; Bao, J.; Li, S.; Lan, Y.; Dai, Z. Polypyrrole -polyoxometalate/reduced graphene oxide ternary nanohybrids for flexible, all-solid-state super capacitors. Chem. Commun. 2015, 51, 12377-12380.

12. Ding, Y.; Peng, J.; Lu, H.; Yuan, Y.; Khan, S. Tungsten addenda mixed heteropolymolybdates supported on functionalized graphene for high-performance aqueous supercapacitors. RSC Adv. 2016, 6, 81085-81091.

13. Genovese, M.; Lian, K. Polyoxometalate modified pine cone biochar carbon for supercapacitor electrodes. J. Mater. Chem. A, 2017, 5, 3939-3947.

14. Chen, H. Y.; Wee, G.; Al-Oweini, R.; Friedl, J.; Tan, K. S.; Wang, Y. X.; Wong, C. L.; Kortz, U.; Stimming, U.; Srinivasan, M. A Polyoxovanadate as an Advanced Electrode Material for Supercapacitors. ChemPhysChem, 2014, 15, 2162-2169.

15. Ruiz, V.; Suarez-Guevara, J.; Gomez-Romero, P. Organic-Inorganic Hybrid Materials for Supercapacitors. ECS Transactions, 2013, 50, 117-123. 
16. Yang, M.; Choi, B.-G.; Jung, S.-C.; Han, Y.; Huh, Y.-S.; Lee, S.-B. Polyoxometalate-coupled Graphene via Polymeric Ionic Liquid Linker for Supercapacitors. Adv. Funct. Mater. 2014, 24, 7301-7309.

17. Wang, G.; Chen, T.; Wang, X.; Ma, H.; Pang, H. High-Performance Supercapacitor Afforded by a High-Connected Keggin-Based 3D Coordination Polymer. Eur. J. Inorg. Chem. 2017, 5350-5355.

18. Wang, G. N.; Chen, T. T.; Li, S. B.; Pang, H. J.; Ma, H. Y. A coordination polymer based on dinuclear (pyrazinyl tetrazolate) copper(II) cations and Wells-Dawson anions for high-performance supercapacitor electrodes. Dalton Trans. 2017, 46, 13897-13902.

19. Lu, L.; Xie, Y. Fabrication and supercapacitor behavior of phosphomolybdicacid/polyaniline/titanium nitride core-shell nanowire array. New J. Chem. 2017, 41, 335-346.

20. Hosseinian, A.; Amjad, A. H.; Hosseinzadeh-Khanmiri, R.; Ghorbani-Kalhor, E.; Babazadeh, M.; Vessally, E. Nanocomposite of ZIF-67 metal-organic framework with reduced graphene oxide nanosheets for high-performance supercapacitor applications. J. Mater. Sci.: Mater. Electron. 2017, 28, 18040-18048.

21. Ajdari, F. B.; Kowsari, E.; Ehsani, A. P-type conductive polymer/zeoliticimidazolate framework-67 (ZIF-67) nanocomposite film: Synthesis, characterization, and electrochemical performance as efficient 
electrode materials in pseudocapacitors. J. Colloid Interface Sci. 2018, 509, 189-194.

22. Qu, C.; Jiao, Y.; Zhao, B.; Chen, D.; Zou, R.; Walton, K. S.; Liu, M. Nickel-based pillared MOFs for high-performance supercapacitors: Design, synthesis and stability study. Nano Energy, 2016, 26, 66-73.

23. Lee, D. Y.; Yoon, S. J.; Shrestha, N. K.; Lee, S. H.; Ahn, H.; Han, S. H. Unusual energy storage and charge retention in Co-based metal-organic-frameworks. Microporous Mesoporous Mater. 2012, 153, 163-165.

24. Diaz, R.; Orcajo, Botas, M. G.; J. A.; Calleja, G.; Palma, J. Co8-MOF-5 as electrode for supercapacitors. Mater. Lett. 2012, 68, 126-128.

25. Lee, D. Y.; Shinde, D. V.; Kim, E. K.; Lee, W.; Oh, I. W.; Shrestha, N. K.; Lee, J. K.; Han, S. H. Supercapacitive property of metal-organic-frameworks with different pore dimensions and morphology. Microporous Mesoporous Mater. 2013, $171,53-57$.

26. Sheberla, D.; Bachman, J. C.; Elias, J. S.; Sun, C. J.; Shao-Horn, Y.; Dincă, M. Conductive MOF electrodes for stable supercapacitors with high areal capacitance. Nature Mater. 2017, 16, 220-224.

27. Wang, L.; Feng, X.; Ren, L.; Piao, Q.; Zhong, J.; Wang, Y.; Li, H.; Chen, Y.; Wang, B. Flexible Solid-State Supercapacitor Based on a Metal-Organic 
Framework Interwoven by Electrochemically-Deposited PANI. J. Am. Chem. Soc. 2015, 137, 4920-4923.

28. Wang, L.; Yang, H.; Pan, G.; Miao, L.; Chen, S.; Song Y., olyaniline-Carbon Nanotubes@Zeolite Imidazolate Framework 67-Carbon Cloth Hierarchical Nanostructures for Supercapacitor Electrode. Electrochim. Acta, 2017, 240, $16-23$.

29. Xu, X.; Tang, J.; Qian, H.; Hou, S.; Bando, Y.; Shahriar, M.; Hossain, A.; Pan, L.; Yamauchi, Y. Three-Dimensional Networked Metal - Organic Frameworks with Conductive Polypyrrole Tubes for Flexible Supercapacitors. ACS Appl. Mater. Interfaces, 2017, 9, 38737-38744.

30. Kang, L.; Sun, S. X.; Kong, L. B.; Lang, J. W.; Luo, Y. C. Investigating metal-organic framework as a new pseudo-capacitive material for supercapacitors. Chin. Chem. Lett. 2014, 25, 957-961.

31. Srimuk, P.; Luanwuthi, S.; Krittayavathananon, A.; Sawangphruk, M. Solid-type supercapacitor of reduced graphene oxide-metal organic framework composite coated on carbon fiber paper. Electrochim. Acta, 2015, 157, 69-77.

32. Gong, Y.; Li, J.; Jiang, P. G.; Li, Q. F.; Lin, J. H. Novel metal(II) coordination polymers based on N,N' -bis-(4-pyridyl)phthalamide as supercapacitor electrode materials in an aqueous electrolyte. Dalton Trans. 2013, 42, 1603-1611. 
33. Guo, S. N.; Zhu, Y.; Yan, Y. Y.; Min, Y. L.; Fan, J. C.; Xu, Q. J.; Jun, H. (Metal-Organic Framework)-Polyaniline sandwich structure composites as novel hybrid electrode materials for high-performance supercapacitor. J. Power Source 2016, 316, 176-182.

34. Saraf, M.; Rajak, R.; Mobin, S. M. A fascinating multitasking Cu-MOF/rGO hybrid for high performance supercapacitors and highly sensitive and selective electrochemical nitrite sensors. J. Mater. Chem. A, 2016, 4, 16432-16445.

35. Wang, X.; Chang, Z.; Lin, H.; Tian, A.; Liu, G.; Zhang, J.; Liu, D. Effect of polyoxoanions and amide group coordination modes on the assembly of polyoxometalate-based metal-organic complexes constructed from a semi-rigid bis-pyridyl-bis-amide ligand. CrystEngComm, 2015, 17, 895-903.

36. Wang, X.; Cao, J.; Liu, G.; Tian, A.; Luan, J.; Lin, H.; Zhang, J.; Li, N. Keggin-based 3D frameworks tuned by silver polymeric motifs: effect of the bi(triazole) substituent group on the architectures. CrystEngComm, 2014, 16, $5732-5740$.

37. Wang, X.; Li, T.; Tian, A. Introduction of secondary pyridyl-1H-tetrazole derivatives into Keggin-Ag-(1,10-phenanthroline) system for tuning dimensionalities and architectures: assembly and properties. J. Coord. Chem. 2016, 69, 2532-2544 
38. Tian, A.; Ji, X.; Xiao, R.; Ni, H.; Tian, Y.; Liu, G.; Ying, J. A series of Keggin-based AgI-belt/cycle structures constructed from 5-phenyl-1H-tetrazole and its derivative through $\mathrm{Ag}-\mathrm{N}$ and $\mathrm{Ag}-\mathrm{C}$ bonds. J. Coord. Chem. 2017, 70, $404-416$

39. Hao, X.; Ma, Y.; Wang, Y.; Xu, L.; Liu, F.; Zhang, M.; Li, Y. New Entangled Coordination Networks Based on Charge-Tunable Keggin- Type Polyoxometalates. Chem. Asian J. 2014, 819-829.

40. 40. Tian, A.; Hou, X.; Ying, J.; Liu, G.; Ning, Y.; Li, T.; Wang, X. Using imdazole-2-carboxylic and biimdazole-4,40-dicarboxylic acid to construct Keggin-based mono-nuclear $\mathrm{Ag}^{+}$subunit and double $\mathrm{Cl}$-capped tri-nuclear $\mathrm{Cu}^{+}$ clusters. Inorg. Chim. Acta, 2016, 439, 43-48.

41. Zhang, Z.; Ma, H.; Pang, H.; Zhang, C.; Chai, D.; Hou, Y. A Zn-tib porous framework sandwiched with Keggin Synthesis, structure, photocatalytic and luminescent properties. J. Solid State Chem. 2018, 258, 170-175.

42. He, Z.; Ma, D.; Cao, B.; Li, X.; Lu, Y. Series of polyoxometalates-based metal-organic frameworks exhibiting high photocatalytic activities for the degradation of methylene blue. Inorg. Chim. Acta, 2018, 471, 316-325.

43. Zhao, X.; Zhang, S.; Yan, J.; Li, L.; Wu, G.; Shi, W.; Yang, G.; Guan, N.; Cheng, P. Polyoxometalate-Based Metal-Organic Frameworks as Visible-Light-Induced Photocatalysts. Inorg. Chem. 2018, 57, 5030-5037. 
44. Jia, S.; Hao, X.; Ma, Y.; Wang, H.; Li, Y. Two new Keggin-type polyoxometalate-based organic-inorganic hybrid compounds with metal ions and chelate ligands. J. Coord. Chem. 2017, 70, 1156-1167.

45. Wang, S.; Yu, K.; Wang, B.; Wang, L.; Wang, C.; Zhang, H.; Wang, C.; Zhou, B. Construction of two novel borotungstates modified by different ligands connected with single/double bridges. New J.Chem. 2016, 40, 7011-7017. 